\title{
CCA Security for Self-Updatable Encryption: Protecting Cloud Data When Clients Read/Write Ciphertexts
}

\author{
Kwangsu Lee* Dong Hoon Lee $\quad$ Jong Hwan Park ${ }^{\ddagger} \quad$ Moti Yung ${ }^{\S}$
}

\begin{abstract}
Self-updatable encryption (SUE) is a new kind of public-key encryption, motivated by cloud computing, which enables anyone (i.e. cloud server with no access to private keys) to update a past ciphertext to a future ciphertext by using a public key. The main applications of SUE is revocable-storage attributebased encryption (RS-ABE) that provides an efficient and secure access control to encrypted data stored in cloud storage. In this setting, there is a new threat such that a revoked user still can access past ciphertexts given to him by a storage server. RS-ABE solves this problem by combining user revocation and ciphertext updating functionalities. The mechanism was designed with semantic security (CPA).

Here, we propose the first SUE and RS-ABE schemes, secure against a relevant form of chosenciphertext security (CCA). Due to the fact that some ciphertexts are easily derived from others, we employ a different notion of CCA which avoids easy challenge related messages. Specifically, we define "time extended challenge" (TEC) CCA security for SUE which excludes ciphertexts that are easily derived from the challenge (over time periods) from being queried on (namely, once a challenge is decided by an adversary, no easy modification of this challenge to future and past time periods is allowed to be queried upon). We then propose an efficient SUE scheme with such CCA security, and we also define similar CCA security for RS-ABE and present an RS-ABE scheme with this CCA security.
\end{abstract}

Keywords: Public-key encryption, Self-updatable encryption, Chosen-ciphertext security, Cloud storage.

* Sejong University, Seoul, Korea. Email: kwangsu@ se jong.ac.kr.

${ }^{\dagger}$ Korea University, Seoul, Korea. Email: donghlee@korea. ac.kr.

${ }^{\ddagger}$ Sangmyung University, Seoul, Korea. Email: jhpark@ smu . ac. kr.

${ }^{\S}$ Snapchat Inc. and Columbia University, New York, USA. Email: moti@ cs . col umbia. edu. 


\section{Introduction}

In cloud storage, providing efficient access control to encrypted data is very important issue, since it extends traditional data in organizations and services to the Internet based hosting paradigm. To provide simple access control, sensitive data may be kept as the encrypted form of attribute based encryption (ABE) with user revocation [4] in cloud storage which is controlled by some servers which do not have access to keys nor are necessarily fully trusted (and may be accessible at times to some clients). Then, there could be potential threats that are new to this setting, as nicely pointed out by Sahai et al. [30]. That is, a user who is revoked from cloud storage may access old ciphertexts (generated before revocation) stored in cloud storage by colluding with insiders, even if he cannot access new ciphertexts generated after revocation. To provide stronger access control to encrypted data stored in cloud storage, revocable-storage attribute-based encryption (RS-ABE) was introduced by Sahai, Seyalioglu, and Waters [30]. RS-ABE, which is a kind of $\mathrm{ABE}$ [17,31], can solve this problem by providing two functionalities: user revocation and ciphertext updating. That is, the access to an old ciphertext with time $T_{1}$ by a revoked user having an old private key with time $T_{2}$ can be prevented if a cloud sever updates the old ciphertext to new one with time $T_{3}$ since the old private key is not enough to decrypt the new ciphertext where $T_{1}<T_{2}<T_{3}$.

Self-updatable encryption (SUE) is a new kind of public-key encryption (PKE) that realizes a timeevolution mechanism by allowing anyone (and storage servers in particular) to update a past ciphertext to a future ciphertext by using public keys (or public parameters). In SUE, a ciphertext and a private key are associated with time $T_{c}$ and $T_{k}$ respectively and the ciphertext can be decrypted by the private key if $T_{c} \leq T_{k}$. Lee, Choi, Lee, Park, and Yung [22] introduced the concept of SUE and proposed an efficient SUE scheme in bilinear groups by utilizing the properties of full binary trees. They also showed that an efficient RS-ABE scheme can be built by combining an SUE scheme and an ABE scheme. That is, the initial RS-ABE scheme of Sahai et al. [30] contains $O\left(\log ^{2} T_{\max }\right)$ group elements in a ciphertext to support ciphertext updating where $T_{\max }$ is the maximum number of time units, while the modularly built RS-ABE scheme of Lee et al. [22] just contains $O\left(\log T_{\max }\right)$ group elements in a ciphertext to support ciphertext updating (due to the use of an SUE scheme). As pointed in [22], SUE can also be used to build time-released encryption (TIE) and key-insulated encryption (KIE) with certain properties.

The available RS-ABE schemes and SUE schemes only provide security (confidentiality) against chosenplaintext attacks (CPA security) [21,22, 30]. CPA security provides sufficient security only for passive attackers who do not modify ciphertexts. Cloud storage, however, allows multiple users to access stored ciphertexts, and insiders who are managing cloud storage can also access these ciphertexts [19, 20]. For example, consider the situation where electronic medical records of patients are stored in cloud storage and these records can be accessed by doctors for a diagnosis or prescription. Using the RS-ABE scheme described above, a patient creates a ciphertext by attaching a policy for accessing his/her medical data, and a doctor who retrieved a private key for his/her own attribute can decrypts the ciphertext if the attributes in the private key satisfy the policy in the ciphertext. In addition, it is possible to flexibly handle the access to these ciphertexts using key revocation and ciphertext updating. In this case, the stored ciphertexts in the cloud storage can be modified by some users (patients, doctors, or administrators) and this modified ciphertext can be decrypted by a doctor if some medical service program is running on this cloud system.

Thus it is needed to guarantee stronger security (confidentiality and integrity) for these ciphertexts even if they are modified due to external malicious users, internal malicious administrators, or unintended system errors. In order to deal with such stronger attackers, it is necessary to consider a stronger security model that considers active attackers instead of conventional passive attackers. For this reason, we consider security against chosen-ciphertext attacks (CCA security), in which an adversary can adaptively request de- 
cryption queries on ciphertexts. CCA security for PKE and its extensions was intensively studied by many researchers and is a standard requirement by now e.g., [1, 12, 13, 26, 28, 34]. In addition, there are general methods to achieve it [6,7, 9]. However, constructing a CCA secure RS-ABE scheme (or CCA secure SUE scheme) is paradoxical by definition, since CCA means non-malleability, but these schemes need to support ciphertext updating functionality over time periods. Thus it seems an unusually hard requirement in this case. Nevertheless, we ask whether and to what extent it is possible to construct some (properly adapted) level of CCA-security for SUE and RS-ABE schemes.

\subsection{Our Results}

In this paper, we first define time extended challenge (TEC) CCA security for SUE by relaxing standard CCA security. In a standard CCA security model, an adversary given a challenge ciphertext is not allowed to probe on it when accessing the decryption oracle to prevent trivial attacks [28]. As mentioned before, the standard CCA security cannot be used for SUE since it supports the ciphertext updating functionality. We extend the restriction of standard CCA security by not allowing the adversary to ask a decryption query on any ciphertext that is the challenge or updated from the challenge ciphertext. This is a natural extension preventing trivial attacks due to the probing capabilities, but leaving non-trivial attacks scenario in place; it is also in the spirit of similar limitations elsewhere [1,12,34]. We then propose an efficient SUE scheme that provides TEC-CCA security under the decisional Bilinear Diffie-Hellman (DBDH) assumption by modifying the SUE scheme of Lee [21]. The design idea of our SUE scheme is given in the later part of this section.

Next, we similarly define TEC-CCA security for RS-ABE since it supports the ciphertext updating functionality as well. We then propose an efficient RS-ABE scheme by combining our TEC-CCA secure SUE scheme, a CCA secure ciphertext-policy ABE (CP-ABE) scheme derived from the CP-ABE scheme of Rouselakis and Waters [29], and the complete subtree (CS) scheme of Naor et al. [25]. We basically follow the design principle of Lee et al. [22] to build our RS-ABE scheme from building blocks, but we propose a key encapsulation mechanism (KEM) of RS-ABE. Additionally, our RS-ABE scheme supports public verifiability of ciphertexts that allows for anyone to check whether ciphertexts are legitimate (original or legally updated) or not. Note that integrity of ciphertexts is easily obtained from public verifiability. We prove that our RS-ABE scheme achieves TEC-CCA security in a selective security model described next. Our RS-ABE scheme is the first one that achieves security beyond CPA, and gives an answer to the question raised by Sahai et al. [30].

The selective revocation list model, introduced by Boldyreva et al. [4], was used in proving the CPA security of revocable $\mathrm{ABE}$ (R-ABE) and RS-ABE if the partitioning technique is employed in the proof. Note that the selective revocation list model in which an adversary should submit a revocation list before receiving public parameters is weaker than the well-known selective model that is used for the security proof of identity-based encryption (IBE) [5, 10] and ABE [17, 31, 35]. In the security proof of our RS-ABE scheme, we show that the TEC-CCA security of our RS-ABE scheme can be proven in the selective TECCCA model instead of the selective revocation list TEC-CCA model although the partitioning technique is used. We note that as a result of independent interest, our new proof technique can also be used to prove the selective CPA security of the R-ABE scheme of Boldyreva et al. [4] instead of the selective revocation list CPA security. 


\subsection{Our Techniques}

A naive approach for building a CCA secure SUE scheme is to employ the CHK method of Canetti et al. [9] by combining a CPA secure SUE scheme of Lee [21] augmented by the Boneh-Boyen IBE scheme [5] and a one-time signature scheme. To improve the efficiency, we use the BMW method of Boyen et al. [7] that is a variant of the CHK method since the role of one-time signature can be replaced by the ciphertext of the BB-IBE scheme and the SUE scheme is a key-encapsulation mechanism (KEM). In this approach, the IBE scheme enables the simulation of the decryption oracle and the one-time signature scheme provides integrity of ciphertexts. However, the resulting CCA secure SUE scheme cannot provide ciphertext updating any longer since the one-time signature scheme is unforgeable. Let $\left(C_{0}=g^{t}, C_{1}=w^{t} \prod v^{s_{i}},\left\{C_{i, 1}=g^{s_{i}}, C_{i, 2}=\right.\right.$ $\left.\left.F_{i, L[i]}\left(\left.L\right|_{i}\right)^{s_{i}}\right\}\right)$ be a CPA secure (simple) SUE ciphertext header and $e(g, g)^{\beta t}$ be a session key. To solve the problem mentioned before, we first divide the ciphertext components of a CPA secure SUE scheme into two parts: the first part $\left(C_{0}=g^{t}\right)$ is related to a session key and the second part $\left(C_{1}=w^{t} \prod v^{S_{i}},\left\{C_{i, 1}, C_{i, 2}\right\}\right)$ is related to ciphertext updating. We then apply the BMW method only to the first part of ciphertext components by adding $C_{3}=\left(u_{S}^{\pi} h_{S}\right)^{t}$ where $\pi=H\left(C_{0}\right)$. The simulation of the decryption oracle and the ciphertext integrity of the first part are achieved by the BMW method. The ciphertext updating functionality in the second part is still preserved, but it is needed to verify that the second part components are correctly updated or not. To provide the ciphertext verifiability of the second part, we observe that the well-formedness of these components can be checked by bilinear maps since these components consist of Diffie-Hellman tuples. By using these techniques, we can build an SUE scheme with TEC-CCA security.

As mentioned before, we combine a TEC-CCA secure SUE scheme, a CCA secure CP-ABE scheme, and the CS scheme to build a TEC-CCA secure RS-ABE scheme motivated by the design principle of Lee et al. [22]. To prove the security of our RS-ABE scheme, we use the well-known partitioning method. However, we need the selective revocation list model to prove the security of RS-ABE as pointed by Lee [21]. The reason is that an adversary can request many private key queries that match to the challenge ciphertext in RS-ABE and these (matching) private keys should be placed on (fixed) leaf nodes in a binary tree for consistency. The selective revocation list model, introduced by Boldyreva et al. [4], is weaker than the well-known selective model [10]. To prove the security of our RS-ABE scheme in the selective model instead of the selective revocation list model, we observe that if a simulator can predict the number of private key queries that match the challenge ciphertext then the problem can be solved by placing a user's private key in a random leaf node of the binary tree. That is, if $\tilde{q}$ is the number of private keys such as $S \in \mathbb{A}^{*}$ where $S$ is the set of attributes in a private key and $\mathbb{A}^{*}$ is the access structure in the challenge ciphertext, then the simulator can fix the positions of these private keys by arbitrary selecting $\tilde{q}$ number of leaf nodes randomly. In this case, the consistency of private keys and update keys is preserved.

\subsection{Related Work}

Cloud Storage. Cloud storage provides data storage services with high availability, easy accessibility, and affordable cost to clients who can not maintain their own independent storage. Cloud storage should provide at least confidentiality and integrity in order to ensure the security of users' data. Classic cloud storage that provides these two properties is a cryptographic file system [16,18]. To provide confidentiality and integrity, this system takes a method of encrypting data with symmetric key encryption and signing data with public key signature when the client stores data in the storage. This method can provide strong security and simple access control functions by providing additional key management and key revocation methods [2], but it is difficult for the cloud server to perform additional work on the encrypted data. In addition to confidentiality and integrity, recent cloud storage additionally provides search (or query) on encrypted data and large file 
integrity verification [19,20]. These cloud storage systems use searchable symmetric key encryption to support searching on encrypted data and proof-of-possession protocols to verify the integrity of large files.

Revocable IBE and Its Extensions. Providing an efficient user revocation in identity-based encryption (IBE) and attribute-based encryption (ABE) is very important issue in real applications. A scalable and efficient revocable IBE (R-IBE) scheme was first proposed by Boldyreva et al. [4] by using a fuzzy IBE scheme and a full binary tree. After that numerous R-IBE schemes were presented in [23,24,27,32,33]. An efficient revocable ABE (R-ABE) scheme also proposed in [4] and its security was claimed in the weaker selective revocation list model. Sahai et al. [30] introduced the concept of RS-ABE to provide efficient access control on encrypted data stored in cloud storage. After the introduction of RS-ABE, an efficient RSABE schemes that incorporate SUE schemes were presented in [21,22]. Lee et al. [22] introduced the notion of SUE and proposed an efficient SUE scheme with CPA security by modifying a hierarchical identity-based encryption (HIBE) scheme [5] to apply the design strategy of forward-secure encryption (FSE) [10]. Then, a number of different SUE schemes were proposed in [14,21]. The main application of SUE is RS-ABE for cloud storage [22]. However, SUE itself can be used for other interesting applications: timed-release encryption and key-insulated encryption.

Chosen-Ciphertext Security. Security against (adaptively) chosen ciphertext attacks (or CCA security) is the standard notion of PKE [28]. For some applications, more adversary constrained notions of CCA security are considered since CCA security is too strong and immediately un-achievable. Such constrained adversary notions have been used before: Dolev et al. introduced nonmalleable security [15], Shoup introduced benign malleability [34], An et al. introduced generalized CCA security (or gCCA security) [1], and Canetti et al. introduced Replayable CCA security (or RCCA security) [12]. Note that benign malleability, gCCA security, and publicly detectable RCCA security are in fact the same notion. This (more adversarially constrained) CCA security was used to prove the security of other cryptographic primitives [11,36].

\section{Preliminaries}

In this section, we define full binary trees and bilinear groups, and then introduce complexity assumptions in bilinear groups.

\subsection{Full Binary Tree}

For binary trees, we follow the notation in [22]. A full binary tree $\mathcal{B} \mathcal{T}$ is a tree data structure where each node except the leaf nodes has two child nodes. Let $N$ be the number of leaf nodes in $\mathcal{B} \mathcal{T}$. The number of all nodes in $\mathcal{B} \mathcal{T}$ is $2 N-1$. For any index $1 \leq i \leq 2 N-1$, we denote by $v_{i}$ a node in $\mathcal{B} \mathcal{T}$. The depth of a node $v_{i}$ is the length of the path from the root node to the node. The root node is at depth zero. The depth of $\mathcal{B} \mathcal{T}$ is the maximum depth of a leaf node. A level of $\mathcal{B} \mathcal{T}$ is a set of all nodes at given depth. Siblings are nodes that share the same parent node.

For each node $v_{i} \in \mathcal{B} \mathcal{T}$, we associated $v_{i}$ with a unique label string $L \in\{0,1\}^{*}$. The label of each node is assigned as follows: Each edge in the tree is assigned with 0 or 1 depending on whether the edge is connected to its left or right child node. The label $L$ of a node $v_{i}$ is defined as the bit string obtained by reading all the labels of edges in the path from the root node to the node $v_{i}$. We assign a special empty string to the root node label. We define $L(i)$ be a mapping from the index $i$ of a node $v_{i}$ to a label $L$. We also use $L\left(v_{i}\right)$ as $L(i)$ if there is no ambiguity. For a label string $L \in\{0,1\}^{\leq n}$, we define some notations: $L[i]$ is the $i$ th bit of $L,\left.L\right|_{i}$ is the prefix of $L$ with $i$-bit length, and $L \| L^{\prime}$ is the concatenation of two strings $L$ and $L^{\prime}$. 
For a full binary tree $\mathcal{B T}$ and a subset $R$ of leaf nodes, $S T(\mathcal{B T}, R)$ is defined as the Steiner Tree induced by the set $R$ and the root node, that is, the minimal subtree of $\mathcal{B} \mathcal{T}$ that connects all the leaf nodes in $R$ and the root node. We simply denote $S T(\mathcal{B T}, R)$ by $S T(R)$.

\subsection{Bilinear Groups}

Let $\mathbb{G}$ and $\mathbb{G}_{T}$ be two multiplicative cyclic groups of prime order $p$. Let $g$ be a generator of $\mathbb{G}$. The bilinear map is a map $e: \mathbb{G} \times \mathbb{G} \rightarrow \mathbb{G}_{T}$ with the following properties:

1. Bilinearity: for all $u, v \in \mathbb{G}$ and all $a, b \in \mathbb{Z}_{p}$, we have $e\left(u^{a}, v^{b}\right)=e(u, v)^{a b}$.

2. Non-degeneracy: $e(g, g) \neq 1$.

We say that $\mathbb{G}$ is a bilinear group if the group operations in $\mathbb{G}$ and $\mathbb{G}_{T}$ as well as the bilinear map $e$ are all efficiently computable.

\subsection{Complexity Assumptions}

Assumption 1 (Decisional Bilinear Diffie-Hellman, DBDH). Let $\left(p, \mathbb{G}, \mathbb{G}_{T}, e\right)$ be a description of the bilinear group of prime order $p$. Let $g$ be a random generator of $\mathbb{G}$. The DBDH assumption is that if the challenge tuple

$$
D=\left(\left(p, \mathbb{G}, \mathbb{G}_{T}, e\right), g, g^{a}, g^{b}, g^{c}\right) \text { and } Z,
$$

are given, no probabilistic polynomial-time (PPT) algorithm $\mathcal{A}$ can distinguish $Z=Z_{0}=e(g, g)^{a b c}$ from $Z=Z_{1}=e(g, g)^{d}$ with more than a negligible advantage. The advantage of $\mathcal{A}$ is defined as $\operatorname{Adv}_{\mathcal{A}}^{D B D H}(\lambda)=$ $\left|\operatorname{Pr}\left[\mathcal{A}\left(D, Z_{0}\right)=0\right]-\operatorname{Pr}\left[\mathcal{A}\left(D, Z_{1}\right)=0\right]\right|$ where the probability is taken over random choices of $a, b, c, d \in \mathbb{Z}_{p}$.

\section{Self-Updatable Encryption}

In this section, we first define the syntax and the chosen ciphertext security of SUE. After that we propose an efficient SUE scheme in bilinear groups and prove its security under the standard assumption.

\subsection{Definitions}

Before we introduce SUE, we define ciphertext delegatable encryption (CDE) that is a building block of SUE. The concept of CDE was introduced by Lee et al. [22] and it is public-key encryption (PKE) that supports the delegation of ciphertexts. In CDE, a ciphertext header is associated with a label string $L$ and a private key is also associated with a label string $L^{\prime}$. The ciphertext header with $L$ can be delegated to a new ciphertext header with a new label string $L^{\prime}$ with the restriction that $L$ is a prefix of $L^{\prime}$. If $L$ is a prefix of $L^{\prime}$, then the ciphertext header with $L$ can be decrypted by the private key with $L^{\prime}$. We slightly modify the definition of CDE in [22]. The syntax of CDE is given as follows:

Definition 3.1 (Ciphertext Delegatable Encryption). A ciphertext delegatable encryption (CDE) scheme for the set $\mathcal{L}$ of labels consists of eight PPT algorithms, Init, Setup, GenKey, RandKey, Encaps, DelegateCT, VerifyCT, and Decaps, which are defined as follows:

Init $\left(1^{\lambda}\right)$. The initialization algorithm takes as input a security parameter $1^{\lambda}$ and outputs a group description string GDS. 
$\operatorname{Setup}(G D S, \ell)$. The setup algorithm takes as input a group description string $G D S$ and the maximum length $\ell$ of the label strings. It outputs a master key $M K$ and public parameters $P P$.

$\operatorname{GenKey}(L, M K, P P)$. The key generation algorithm takes as input a label string $L \in\{0,1\}^{n}$ with $n \leq \ell$, the master key $M K$, and the public parameters $P P$. It outputs a private key $S K_{L}$.

$\operatorname{RandKey}\left(S K_{L}, \delta, P P\right)$. The key randomization algorithm takes as input a private key $S K_{L}$, an exponent $\delta$, and the public parameters $P P$. It outputs a randomized private key $S K_{L}$.

$\operatorname{Encaps}(L, t, \vec{s}, P P)$. The encapsulation algorithm takes as input a label string $L \in\{0,1\}^{d}$ with $d \leq \ell$, an exponent $t$, an exponent vector $\vec{s}$, and the public parameters $P P$. It outputs a ciphertext header $C H_{L}$ and a session key $E K$.

DelegateCT $\left(\mathrm{CH}_{L}, c, P P\right)$. The ciphertext delegation algorithm takes as input a ciphertext header $\mathrm{CH}_{L}$ for a label string $L \in\{0,1\}^{d}$ with $d<\ell$, a bit value $c \in\{0,1\}$, and the public parameters $P P$. It outputs a delegated ciphertext header $C H_{L^{\prime}}$ for the label string $L^{\prime}=L \| c$.

VerifyCT $\left(\mathrm{CH}_{L}, L, P P\right)$. The ciphertext verification algorithm takes as input a ciphertext header $\mathrm{CH}_{L}$, a label string $L$, and the public parameters $P P$. It outputs 1 if the ciphertext header is valid or 0 otherwise.

Decaps $\left(\mathrm{CH}_{L}, S K_{L^{\prime}}, P P\right)$. The decapsulation algorithm takes as input a ciphertext header $\mathrm{CH}_{L}$, a private key $S K_{L^{\prime}}$, and the public parameters $P P$. It outputs a session key $E K$ or the distinguished symbol $\perp$.

The correctness property of CDE is defined as follows: For all $P P, M K$ generated by Setup, any $S K_{L^{\prime}}$ generated by GenKey, any $C_{L}$ and $E K$ generated by Encaps or DelegateCT, it is required that:

- If $L$ is a prefix of $L^{\prime}$, then Decaps $\left(C H_{L}, S K_{L^{\prime}}, P P\right)=E K$.

- If $L$ is not a prefix of $L^{\prime}$, then Decaps $\left(\mathrm{CH}_{L}, S K_{L^{\prime}}, P P\right)=\perp$ with all but negligible probability.

Additionally, it requires that the delegated ciphertext header of DelegateCT is a valid ciphertext header under the new label string.

SUE, introduced by Lee et al. [22], is new PKE that supports the updating of ciphertexts by using a public key (or public parameters). In SUE, a ciphertext header is associated with time $T$ and a private key is associated with time $T^{\prime}$. If $T^{\prime} \geq T$, then the ciphertext header with $T$ can be decrypted by a private key with $T^{\prime}$. That is, the ciphertext header with $T$ can be updated to a new ciphertext header with $T^{\prime}$ and then the private key with $T^{\prime}$ can decrypt the updated ciphertext header. We slightly modify the definition of SUE in [22]. The syntax of SUE is given as follows:

Definition 3.2 (Self-Updatable Encryption). A self-updatable encryption (SUE) scheme consists of eight PPT algorithms, Init, Setup, GenKey, RandKey, Encaps, UpdateCT, VerifyCT, and Decaps, which are defined as follows:

$\operatorname{Init}\left(1^{\lambda}\right)$. The initialization algorithm takes as input a security parameter $1^{\lambda}$ and outputs a group description string $G D S$.

$\operatorname{Setup}\left(G D S, T_{\max }\right)$. The setup algorithm takes as input a group description string $G D S$ and the maximum time $T_{\max }$. It outputs a master key $M K$ and public parameters $P P$.

$\operatorname{GenKey}(T, M K, P P)$. The key generation algorithm takes as input time $T$, the master key $M K$, and the public parameters $P P$. It outputs a private key $S K_{T}$. 
$\operatorname{RandKey}\left(S K_{T}, \delta, P P\right)$. The key randomization algorithm takes as input a private key $S K_{T}$, an exponent $\delta$, and the public parameters $P P$. It outputs a randomized private key $S K_{T}$.

$\operatorname{Encaps}(T, t, P P)$. The encapsulation algorithm takes as input time $T$, an exponent $t$, and the public parameters $P P$. It outputs a ciphertext header $C H_{T}$ and a session key $E K$.

UpdateC $\mathbf{T}\left(\mathrm{CH}_{T}, \mathrm{~T}+1, \mathrm{PP}\right)$. The ciphertext update algorithm takes as input a ciphertext header $\mathrm{CH}_{T}$ for time $T$, next time $T+1$, and the public parameters $P P$. It outputs an updated ciphertext header $C H_{T+1}$.

Verify $\mathbf{C T}\left(\mathrm{CH}_{T}, T, P P\right)$. The ciphertext verification algorithm takes as input a ciphertext header $\mathrm{CH}_{T}$, time $T$, and the public parameters $P P$. It outputs 1 if the ciphertext header is valid or 0 otherwise.

Decaps $\left(\mathrm{CH}_{T}, S K_{T^{\prime}}, P P\right)$. The decapsulation algorithm takes as input a ciphertext header $\mathrm{CH}_{T}$, a private key $S K_{T^{\prime}}$, and the public parameters $P P$. It outputs a session key $E K$ or the distinguished symbol $\perp$.

The correctness property of SUE is defined as follows: For all $P P, M K$ generated by Setup, all $T, T^{\prime}$, any $S K_{T^{\prime}}$ generated by GenKey, and any $C_{T}$ and $E K$ generated by Encaps or UpdateCT, it is required that:

- If $T \leq T^{\prime}$, then $\operatorname{Decaps}\left(C_{T}, S K_{T^{\prime}}, P P\right)=E K$.

- If $T>T^{\prime}$, then Decaps $\left(C H_{T}, S K_{T^{\prime}}, P P\right)=\perp$ with all but negligible probability.

Additionally, it requires that the updated ciphertext header of UpdateCT is a valid ciphertext header under the new time.

Remark 1. The syntax of SUE in [22] additionally includes the ciphertext randomization algorithm RandCT, but we omit this algorithm in the above syntax of SUE. The RandCT algorithm ensures that the ciphertext distribution of UpdateCT is statistically equal to that of Encaps by completely re-randomizing the output of UpdateCT. However, we weaken this strong requirement in this paper by just requiring that the output of UpdateCT is just a valid ciphertext header because of the reason in Remark 4 . In this case, we do not need to completely re-randomize the updated ciphertext header of UpdateCT.

Remark 2. If a ciphertext header $\mathrm{CH}_{T}$ with time $T$ is updated to multiple ciphertext headers $\mathrm{CH}_{T_{1}}, \mathrm{CH}_{T_{2}}$, and $\mathrm{CH}_{T_{3}}$ where $T_{1}, T_{2}, T_{3} \geq T$, then those updated ciphertext headers should be re-randomized to remove the relationship between ciphertext headers. That is, an adversary who obtained the session key of a ciphertext header can use this session key to break other ciphertext headers if they are not re-randomized. However, in most applications we can ensure that a ciphertext header $\mathrm{CH}_{T}$ is updated to a new single ciphertext header $\mathrm{CH}_{T+1}$ and completely delete the previous one. In this case, we can prevent the previous attack since there is only one ciphertext header that is related to the original ciphertext header.

Security against chosen plaintext attacks (CPA security) for SUE was introduced by Lee et al. [22]. We define security against chosen ciphertext attacks (CCA security) for SUE by modifying their definition of CPA security. To be precise, there are several notions of CCA security: security against lunchtime attacks (or CCA1 security) and security against adaptively chosen ciphertext attacks (or CCA2 security) [26, 28]. We simply use CCA security for CCA2 security. Although CCA security is regarded as the standard notion for security of encryption schemes, it is too strong for SUE since CCA security cannot be achievable in SUE. In CCA security, an adversary is allowed to request a decryption query with the restriction that the challenge ciphertext given to the adversary cannot be queried. However, an adversary attacking an SUE scheme can query an updated ciphertext to the decryption oracle after obtaining the updated ciphertext by updating the challenge ciphertext. Thus, the adversary can easily break CCA security of SUE. To solve this problem in 
security definition, we relax the CCA security by restricting that the adversary cannot query the decryption oracle on a ciphertext that is the challenge or updated from the challenge ciphertext. We may view these ciphertexts that are the challenge or updated from the challenge ciphertext as time extended challenge (TEC) ciphertexts. The TEC-CCA security of SUE is given as follows:

Definition 3.3 (Selective TEC-CCA Security). The selective TEC-CCA security for SUE schemes is defined in terms of the indistinguishability under time extended challenge chosen plaintext attacks (IND-TEC-CCA). The security game is defined as the following game between a challenger $\mathcal{C}$ and a PPT adversary $\mathcal{A}$ :

Init: $\mathcal{A}$ first submits the challenge time $T^{*}$.

Setup: $\mathcal{C}$ runs the initialization and setup algorithms to generate $M K$ and $P P$. It gives $P P$ to $\mathcal{A}$.

Query 1: $\mathcal{A}$ adaptively requests a polynomial number of private key and decryption queries. $\mathcal{C}$ handles the queries as follows:

- If this is a private key query for time $T$ subject to the restriction $T<T^{*}$, then it creates the private key $S K_{T}$ for the time $T$ by calling the key generation algorithm. It responses the query with $S K_{T}$ to $\mathcal{A}$.

- If this is a decryption query for a ciphertext header $\mathrm{CH}_{T}$, then it computes the decapsulated session key $E K$ by calling the decryption algorithm and responses the query with $E K$ to $\mathcal{A}$.

Challenge: $\mathcal{A}$ requests a challenge ciphertext header and a challenge session key. $\mathcal{C}$ creates a ciphertext header $C H_{T^{*}}^{*}$ and a session key $E K^{*}$ by calling the encryption algorithm under the challenge time $T^{*}$. It flips a random bit $\gamma \in\{0,1\}$. If $\gamma=0$, then it gives $C H_{T^{*}}^{*}$ and $E K^{*}$ to $\mathcal{A}$. Otherwise, it gives $C H_{T^{*}}^{*}$ and a random session key $E K^{\prime}$ to $\mathcal{A}$. Note that $\mathcal{A}$ can obtain updated ciphertext headers that are derived from the challenge ciphertext header since the ciphertext update algorithm can be performed by anyone.

Query 2: $\mathcal{A}$ continues to request private key and decryption queries. $\mathcal{C}$ handles the private key queries as the same as before. It handles the decryption queries as follows:

- If this is a decryption query for a ciphertext header $\mathrm{CH}_{T}$ subject to the restriction that $\mathrm{CH}_{T}$ is not updated from $C H_{T^{*}}^{*}$ in case of $T \geq T^{*}$, then it computes the decapsulated session key $E K$ by calling the decryption algorithm. It responses the query with $E K$ to $\mathcal{A}$.

Guess: Finally $\mathcal{A}$ outputs a bit $\gamma^{\prime}$.

The advantage of $\mathcal{A}$ is defined as $\operatorname{Adv}_{\mathcal{A}}^{S U E}(\lambda)=\left|\operatorname{Pr}\left[\gamma=\gamma^{\prime}\right]-\frac{1}{2}\right|$ where the probability is taken over all the randomness of the game. An SUE scheme is selectively secure under time extended challenge chosen ciphertext attacks if for all PPT adversaries $\mathcal{A}$, the advantage of $\mathcal{A}$ in the above game is negligible in the security parameter $\lambda$.

Remark 3. The above TEC-CCA security for SUE is closely related to the relaxed CCA security notions: the benign malleability of Shoup [34], the generalized CCA (gCCA) security of An et al. [1], and the public detectable Replayable CCA (RCCA) security of Canetti et al. [12]. In these relaxed CCA security models, there exists an efficiently computable relation $R(-,-)$ on ciphertext headers and the decryption results of two ciphertext headers $\mathrm{CH}_{1}$ and $\mathrm{CH}_{2}$ are equal if $\mathrm{R}\left(\mathrm{CH}_{1}, \mathrm{CH}_{2}\right)=$ True. Thus, the decryption query on any ciphertext header $\mathrm{CH}^{\prime}$ that satisfies $\mathrm{R}\left(\mathrm{CH}^{*}, \mathrm{CH}^{\prime}\right)=$ True where $\mathrm{CH}^{*}$ is the challenge ciphertext header is not allowed in this model. In TEC-CCA, the relation $R(-,-)$ checks whether a ciphertext header is updated from another ciphertext header or not. 
Remark 4. If an SUE scheme can re-randomize the output of the UpdateCT algorithm, then this rerandomizable SUE scheme cannot satisfy the TEC-CCA security. The reason is that there is no efficiently computable relation $R(-,-)$ that can check whether a ciphertext header is updated from the challenge ciphertext header or not. Therefore, the syntax of SUE for TEC-CCA security only requires for the UpdateCT algorithm to output a just valid ciphertext header.

\subsection{Managing the Time Structure}

To efficiently manage the time structure, we use a full binary tree as in [10, 22, 30]. We define some useful notations for a binary tree. Let $v$ be a node in $\mathcal{B} \mathcal{T}$. Parent $(v)$ is the parent node of the input node $v$. $\operatorname{Path}(v)$ is the set of path nodes from the root node to the input node $v$. RightSibling $(v)$ is the right sibling node of $v$. That is, $\operatorname{RightSibling}(v)=\operatorname{RightChild}(\operatorname{Parent}(v))$ where $\operatorname{RightChild}(v)$ is the right child of $v$. We also define $\operatorname{TimeNodes}(v)=\{v\} \cup \operatorname{RightSibling}(\operatorname{Path}(v)) \backslash \operatorname{Path}(\operatorname{Parent}(v))$ where $\operatorname{RightSibling}(\operatorname{Path}(v))$ is the set of right sibling nodes of $\operatorname{Path}(v)$. Let $L$ be the label string of a node $v$. We also define $\operatorname{Parent}(L)$, $\operatorname{Path}(L)$, RightSibling $(L)$, and $\operatorname{TimeNodes}(L)$ similarly except that these are defined by using the label string $L$ of $v$.

For each node in $\mathcal{B T}$, we assign a unique time value $T \in\left\{1, \ldots, T_{\max }\right\}$ by using pre-order traversal that recursively visits the root node, the left subtree, and the right subtree. That is, the root node is assigned to 1 , the left most leaf node is assigned to $d+1$, and the right most leaf node is assigned to $T_{\max }=2^{d+1}-1$ where $d$ is the depth of the tree. We let $v_{T}$ be a node associated with time $T$. We define a mapping $\psi$ from time $T$ to a label $L$. That is, $\psi(T)$ returns the label $L$ of a node $v_{T}$ associated with time $T$. The following theorem guarantees that we can handle the time components efficiently.

Theorem 3.1 ( [30]). Let $\mathcal{B T}$ be a full binary tree of depth $d$ and $v_{T}$ be a node associated with time $T$ by pre-order traversal. For any node $v_{T} \in \mathcal{B} \mathcal{T}$, TimeNodes $\left(v_{T}\right)$ satisfies the following properties:

- Property 1. TimeNodes $\left(v_{T}\right) \cap \boldsymbol{P a t h}\left(v_{T^{\prime}}\right) \neq \emptyset$ if and only if $T \leq T^{\prime}$

- Property 2. If $v \in \operatorname{TimeNodes}\left(v_{T+1}\right)$, then there is an ancestor of $v$ in $\operatorname{TimeNodes}\left(v_{T}\right)$.

- Property 3. $\mid$ TimeNodes $\left(v_{T}\right) \mid \leq d+1$

Remark 5. In pre-order traversal, if $v_{T}$ is an internal node, then $v_{T+1}=\operatorname{LeftChild}\left(v_{T}\right)$. If $v_{T}$ is a leaf node, then $v_{T+1}=\operatorname{RightChild}\left(v_{T^{\prime}}\right)$ where $v_{T^{\prime}} \in \operatorname{Path}\left(v_{T}\right)$ is a node with the largest depth such that $\operatorname{LeftChild}\left(v_{T^{\prime}}\right) \in$ $\operatorname{Path}\left(v_{T}\right)$.

\subsection{Construction}

We use the CPA secure CDE scheme of Lee [21] as the building block of our TEC-CCA secure SUE scheme. The main challenge when devising a TEC-CCA secure SUE scheme is providing the integrity of ciphertexts while the updating of ciphertexts is also provided. To solve this problem, we observe that ciphertext elements can be divided into two parts: one part is related to the ciphertext updating and another part is related to the session key of the ciphertext. The validity of ciphertext elements for the ciphertext updating can be easily checked by using bilinear maps since these elements are composed of Diffie-Hellman (DH) tuples. The integrity of ciphertext elements for the session key can be achieved by using the well-known transformation of Canetti et al. [9]. To be precise, we use the direct method of Boyen et al. [7] to improve the efficiency. The modified CDE scheme of Lee [21] is described as follows: 
CDE.Init $\left(1^{\lambda}\right)$ : It first generates bilinear groups $\mathbb{G}, \mathbb{G}_{T}$ of prime order $p$. It chooses a random generator $g \in \mathbb{G}$ and outputs $G D S=\left(\left(p, \mathbb{G}, \mathbb{G}_{T}, e\right), g\right)$.

$\operatorname{CDE} . \operatorname{Setup}(G D S, \ell)$ : It chooses a random exponent $\beta \in \mathbb{Z}_{p}$ and random elements $w, v, u,\left\{h_{i, 0}, h_{i, 1}\right\}_{i=1}^{\ell} \in \mathbb{G}$. Let $F_{i, b}(L)=u^{L} h_{i, b}$ where $i \in[\ell]$ and $b \in\{0,1\}$. It outputs a master key $M K=\beta$ and public parameters $P P=\left(\left(p, \mathbb{G}, \mathbb{G}_{T}, e\right), g, w, v, u,\left\{h_{i, 0}, h_{i, 1}\right\}_{i=1}^{\ell}, \Lambda=e(g, g)^{\beta}\right)$.

CDE.GenKey $(L, M K, P P)$ : Let $L$ be an $n$-bit label string. It chooses random exponents $r, r_{1}, \ldots, r_{n} \in \mathbb{Z}_{p}$ and outputs a private key $S K_{L}=\left(K_{0}=g^{\beta} w^{r}, K_{1}=g^{-r},\left\{K_{i, 1}=v^{r} F_{i, L[i]}\left(\left.L\right|_{i}\right)^{r_{i}}, K_{i, 2}=g^{-r_{i}}\right\}_{i=1}^{n}\right)$.

CDE.RandKey $\left(S K_{L}, \delta, P P\right)$ : Let $S K_{L}=\left(K_{0}, K_{1},\left\{K_{i, 1}, K_{i, 2}\right\}\right)$ and $\delta$ be an exponent in $\mathbb{Z}_{p}$. It selects random exponents $r^{\prime}, r_{1}^{\prime}, \ldots, r_{n}^{\prime} \in \mathbb{Z}_{p}$ and outputs a re-randomized private key $S K_{L}=\left(K_{0}^{\prime}=K_{0} \cdot g^{\delta} w^{r^{\prime}}, K_{1}^{\prime}=\right.$ $\left.K_{1} \cdot g^{-r^{\prime}},\left\{K_{i, 1}^{\prime}=K_{i, 1} \cdot v^{r^{\prime}} F_{i, L[i]}\left(\left.L\right|_{i}\right)^{r_{i}^{\prime}}, K_{i, 2}^{\prime}=K_{i, 2} \cdot g^{-r_{i}^{\prime}}\right\}_{i=1}^{n}\right)$.

CDE.Encaps $(L, t, \vec{s}, P P)$ : Let $L$ be a $d$-bit label string. By using the given exponent $t \in \mathbb{Z}_{p}$ and the vector $\vec{s}=$ $\left(s_{1}, \ldots, s_{d}\right) \in \mathbb{Z}_{p}^{d}$, it outputs a ciphertext header $C H_{L}=\left(C_{0}=g^{t}, C_{1}=w^{t} \prod_{i=1}^{d} v^{s_{i}},\left\{C_{i, 1}=g^{s_{i}}, C_{i, 2}=\right.\right.$ $\left.\left.F_{i, L[i]}\left(\left.L\right|_{i}\right)^{s_{i}}\right\}_{i=1}^{d}\right)$ and a session key $E K=\Lambda^{t}$.

CDE.DelegateCT $\left(C H_{L}, c, P P\right)$ : Let $C_{L}=\left(C_{0}, C_{1},\left\{C_{i, 1}, C_{i, 2}\right\}\right)$ where $L \in\{0,1\}^{d}$. It first sets a new label string $L^{\prime}=L \| c$ where $c \in\{0,1\}$. It selects a random exponent $s_{d+1} \in \mathbb{Z}_{p}$ and outputs a delegated ciphertext header $C H_{L^{\prime}}=\left(C_{0}^{\prime}=C_{0}, C_{1}^{\prime}=C_{1} \cdot v^{s_{d+1}},\left\{C_{i, 1}^{\prime}=C_{i, 1}, C_{i, 2}^{\prime}=C_{i, 2}\right\}_{i=1}^{d}, C_{d+1,1}^{\prime}=g^{s_{d+1}}, C_{d+1,2}^{\prime}=\right.$ $\left.F_{d+1, c}\left(L^{\prime}\right)^{s_{d+1}}\right)$.

CDE.VerifyCT $\left(\mathrm{CH}_{L}, L, P P\right)$ : Let $\mathrm{CH}_{L}=\left(C_{0}, C_{1},\left\{C_{i, 1}, C_{i, 2}\right\}\right)$ for a label string $L \in\{0,1\}^{d}$. It checks that $e\left(C_{1}, g\right) \stackrel{?}{=} e\left(C_{0}, w\right) \cdot e\left(\prod_{i=1}^{d} C_{i, 1}, v\right)$ and $e\left(C_{i, 2}, g\right) \stackrel{?}{=} e\left(C_{i, 1}, F_{i, L[i]}\left(\left.L\right|_{i}\right)\right)$ for all $i \in\{1, \ldots, d\}$. If all tests pass, then it outputs 1 . Otherwise, it outputs 0 .

CDE.Decaps $\left(C_{L}, S K_{L^{\prime}}, P P\right)$ : Let $C H_{L}=\left(C_{0}, C_{1},\left\{C_{i, 1}, C_{i, 2}\right\}_{i=1}^{d}\right)$ for a label string $L \in\{0,1\}^{d}$ and $S K_{L^{\prime}}=$ $\left(K_{0}, K_{1},\left\{K_{i, 1}, K_{i, 2}\right\}_{i=1}^{n}\right)$ for a label string $L^{\prime} \in\{0,1\}^{n}$. If $L$ is a prefix of $L^{\prime}$, then it outputs a session key $E K$ by computing $e\left(C_{0}, K_{0}\right) \cdot e\left(C_{1}, K_{1}\right) \cdot \prod_{i=1}^{d}\left(e\left(C_{i, 1}, K_{i, 1}\right) \cdot e\left(C_{i, 2}, K_{i, 2}\right)\right)$. Otherwise, it outputs $\perp$.

Remark 6. Compared to the CDE scheme of Lee [21], we added the VerifyCT algorithm and modified the Decaps algorithm. The VerifyCT algorithm simply checks the validity of CDE ciphertext headers by using bilinear maps since the group elements in a CDE ciphertext header consist of DDH tuples. The Decaps algorithm derives a session key without running the DelegateCT algorithm.

Let $\mathcal{H}$ be a family of collision resistant hash functions $H^{z}$, indexed by some finite index set $\{z\}$. Our SUE scheme is described as follows:

SUE.Init( $\left(1^{\lambda}\right)$ : It outputs $G D S$ by running CDE.Init $\left(1^{\lambda}\right)$.

SUE.Setup $\left(G D S, T_{\max }\right)$ : It first chooses random elements $u_{S}, h_{S} \in \mathbb{G}$ and obtains $M K_{C D E}$ and $P P_{C D E}$ by running CDE.Setup $(G D S, \ell)$ where $T_{\max }=2^{\ell+1}-1$. It also chooses a random index $z$ for a hash function $H^{z} \in \mathcal{H}$. It outputs a master key $M K=M K_{C D E}$ and public parameters $P P=\left(P P_{C D E}, z, u_{S}, h_{S}\right)$.

SUE.GenKey $(T, M K, P P)$ : It outputs $S K_{T}$ by running CDE.GenKey $(\psi(T), M K, P P)$.

SUE.RandKey $\left(S K_{T}, \delta, P P\right)$ : It outputs $S K_{T}$ by running $\operatorname{CDE} \cdot \operatorname{RandKey}\left(S K_{T}, \delta, P P\right)$.

SUE.Encaps $(T, t, P P)$ : It sets a label string $L=\psi(T)$ and proceeds as follows: 
1. It first obtains $T L=\left(L^{(0)}, L^{(1)}, \ldots, L^{(d)}\right)$ by computing $\operatorname{TimeNodes}(L)$ where $L=L^{(0)}$. Let $d^{(j)}$ be the length of the label $L^{(j)}$. Note that the labels in $T L$ are ordered according to pre-order traversal.

2. For each label $L^{(j)}$ in $T L$ such that $0 \leq j \leq d$, it proceeds the following steps:

(a) If $j=0$, then it sets a vector $\vec{s}=\left(s_{1}, \ldots, s_{d^{(0)}}\right)$ by selecting random exponents $s_{1}, \ldots, s_{d^{(0)}} \in$ $\mathbb{Z}_{p}$. It obtains $C H^{(0)}=\left(C_{0}, C_{1},\left\{C_{i, 1}, C_{i, 2}\right\}_{i=1}^{d^{(0)}}\right)$ and $E K$ by running $\operatorname{CDE.Encaps}\left(L^{(0)}, t, \vec{s}, P P\right)$.

(b) If $j \geq 1$, then it sets a new vector $\vec{s}^{\prime}=\left(s_{1}^{\prime}, \ldots, s_{d^{(j)}-1}^{\prime}, s_{d^{(j)}}^{\prime}\right)$ where $s_{1}^{\prime}, \ldots s_{d^{(j)}-1}^{\prime}$ are copied from $\vec{s}$ and $s_{d^{(j)}}^{\prime}$ is randomly selected in $\mathbb{Z}_{p}$. It obtains $C H^{(j)}=\left(C_{0}^{\prime}, C_{1}^{\prime},\left\{C_{i, 1}^{\prime}, C_{i, 2}^{\prime}\right\}_{i=1}^{d^{(j)}}\right)$ by running CDE.Encaps $\left(L^{(j)}, t, \vec{s}^{\prime}, P P\right)$. Next, it removes redundant elements $C_{0}^{\prime},\left\{C_{i, 1}^{\prime}, C_{i, 2}^{\prime}\right\}_{i=1}^{d^{(j)}-1}$ from $C H^{(j)}$ since they are contained in $C H^{(0)}$.

3. It computes $\pi=H^{z}\left(C_{0}\right)$ and sets $C_{3}=\left(u_{S}^{\pi} h_{S}\right)^{t}$.

4. It outputs a ciphertext header $\mathrm{CH}_{T}=\left(\mathrm{CH}^{(0)}, C H^{(1)}, \ldots, C H^{(d)}, \mathrm{C}_{3}\right)$ and $E K$.

SUE.UpdateCT $\left(\mathrm{CH}_{T}, T+1, P P\right)$ : Let $\mathrm{CH}_{T}=\left(\mathrm{CH}^{(0)}, \ldots, \mathrm{CH}^{(d)}, \mathrm{C}_{3}\right)$ and $\mathrm{L}^{(j)}$ be the label of $\mathrm{CH}^{(j)}$. It proceeds as follows:

1. If the length $d$ of $L^{(0)}$ is less than $\ell$, then it first obtains $C H_{L^{(0)} \| 0}$ and $C H_{L^{(0)} \| 1}$ by running CDE.DelegateCT $\left(\mathrm{CH}^{(0)}, c, P P\right)$ for all $c \in\{0,1\}$ since $\mathrm{CH}_{L^{(0)} \| 0}$ is the ciphertext for the next time $T+1$ by pre-order traversal. Next, it prunes redundant elements in $C H_{L^{(0)} \| 1}$. It outputs an updated ciphertext header $\mathrm{CH}_{T+1}=\left(\mathrm{CH}^{\prime(0)}=\mathrm{CH}_{L^{(0)} \| 0}, \mathrm{CH}^{\prime(1)}=\mathrm{CH}_{L^{(0)} \| 1}, \mathrm{CH}^{\prime(2)}=\right.$ $\left.\mathrm{CH}^{(1)}, \ldots, \mathrm{CH}^{(d+1)}=\mathrm{CH}^{(d)}, \mathrm{C}_{3}\right)$.

2. Otherwise, it copies common elements in $C H^{(0)}$ to $C H^{(1)}$ and simply removes $C H^{(0)}$ since $C H^{(1)}$ is the ciphertext header for the next time $T+1$ by pre-order traversal. It outputs an updated ciphertext header $\mathrm{CH}_{T+1}=\left(\mathrm{CH}^{\prime(0)}=\mathrm{CH}^{(1)}, \ldots, \mathrm{CH}^{\prime(d-1)}=\mathrm{CH}^{(d)}, \mathrm{C}_{3}\right)$.

SUE.VerifyCT $\left(\mathrm{CH}_{T}, T, P P\right)$ : Let $\mathrm{CH}_{T}=\left(\mathrm{CH}^{(0)}, \ldots, \mathrm{CH}^{(d)}, \mathrm{C}_{3}\right)$. It sets a label string $L=\psi(T)$ and proceeds as follows:

1. It first obtains $T L=\left(L^{(0)}, L^{(1)}, \ldots, L^{(d)}\right)$ by computing TimeNodes $(L)$ where $L=L^{(0)}$. Note that the labels in $T L$ are ordered according to pre-order traversal. It checks that the number of labels in $T L$ is the same as the number of CDE ciphertext headers in $\mathrm{CH}_{T}$.

2. For each label $L^{(j)}$ in $T L$, it checks $1 \stackrel{?}{=}$ CDE.VerifyCT $\left(C H^{(j)}, L^{(j)}, P P\right)$. Note that the common elements of $C H^{(0)}$ should be copied to $C H^{(j)}$ when $j>0$.

3. Next, it computes $\pi=H^{z}\left(C_{0}\right)$ and checks $e\left(C_{3}, g\right) \stackrel{?}{=} e\left(C_{0}, u_{S}^{\pi} h_{S}\right)$ where $C_{0}$ is the element in $\mathrm{CH}^{(0)}$.

4. It outputs 1 if all checking pass. Otherwise, it outputs 0.

SUE.Decaps $\left(\mathrm{CH}_{T}, S K_{T^{\prime}}, P P\right)$ : If $T>T^{\prime}$, then it outputs $\perp$ since it cannot decrypt. Otherwise, it proceeds as follows: It first checks $1 \stackrel{?}{=} \mathbf{S U E . V e r i f y C T}\left(\mathrm{CH}_{T}, T, P P\right)$. If the checking fails, then it outputs $\perp$. Next, it finds $C H^{(j)}$ from $C H_{T}$ such that $L^{(j)}$ is a prefix of $L^{\prime}=\psi\left(T^{\prime}\right)$ and outputs $E K$ by running CDE.Decaps $\left(\mathrm{CH}^{(j)}, S K_{T^{\prime}}, P P\right)$. 
Remark 7. Compared to the SUE scheme of Lee [21], the Encaps and Decaps algorithms of our SUE scheme are different. The Encaps algorithm additionally generates a group element $C_{3}$ to provide the integrity of the group element $C_{0}$ by using the direct method of Boyen et al. [7]. The Decaps algorithm checks the validity of the ciphertext header before it derives a session key by running the VerifyCT algorithm. The VerifyCT algorithm first checks the validity of CDE ciphertext headers by running CDE.VerifyCT and then checks the integrity of $C_{0}$ by using bilinear maps.

Remark 8. Let $\mathrm{CH}_{T}$ and $\mathrm{CH}_{T^{\prime}}^{\prime}$ be two valid SUE ciphertext headers with group elements $C_{0}$ and $C_{0}^{\prime}$ respectively. The efficiently computable relation $\mathrm{R}\left(\mathrm{CH}_{T}, \mathrm{CH}_{T^{\prime}}^{\prime}\right)$ in Remark 3 returns $\mathrm{True}_{\mathrm{i}} \mathrm{CH}_{T}$ and $\mathrm{CH}_{T^{\prime}}^{\prime}$ are valid SUE ciphertext headers, $T \leq T^{\prime}$, and $C_{0}=C_{0}^{\prime}$ since the integrity of $C_{0}$ and $C_{0}^{\prime}$ is preserved after running the ciphertext update algorithm.

\subsection{Correctness}

The correctness of the CDE scheme was shown by Lee [21]. Note that the modified Decaps algorithm preserve the correctness. Let $C H_{T}$ be a ciphertext header with time $T$ generated by SUE.Encaps and $S K_{T^{\prime}}$ be a private key with time $T^{\prime}$ generated by SUE.GenKey. To show the correctness of the above SUE scheme, we should show that SUE.Decaps derives a valid session key $E K$ from $C H_{T}$ and $S K_{T^{\prime}}$ if $T \leq T^{\prime}$ and that $\mathrm{CH}_{T+1}$ generated by SUE.UpdateCT is a valid ciphertext header with time $T+1$.

We first show that SUE.Decaps can derive a valid session key by presenting that SUE.VerifyCT can check the validity of $\mathrm{CH}_{T}$ and CDE.Decaps can be used to derive a session key. The algorithm SUE.VerifyCT checks that the number of $\mathrm{CDE}$ ciphertext headers in $\mathrm{CH}_{T}$, the validity of each $\mathrm{CDE}$ ciphertext header by running CDE.VerifyCT, and the validity of $C_{3}$. The validity of $\mathrm{CDE}$ ciphertext headers can be easily checked by using bilinear maps since a CDE ciphertext header consists of elements composed of DiffieHellman (DH) tuples and bilinear maps can check the validity of DDH tuples. The validity of $C_{3}$ also can be easily checked by using bilinear maps since $\left(C_{0}, u_{S}^{\pi} h_{s}, C_{3}\right)$ is also a DDH tuple.

The CDE.Decaps algorithm only can derive a valid session key if the label of a CDE ciphertext header is a prefix of the label of a CDE private key. From the property 2 of Theorem 3.1, we have TimeNodes $(\psi(T)) \cap$ $\operatorname{Path}\left(\psi\left(T^{\prime}\right)\right) \neq \emptyset$ if $T \geq T^{\prime}$ where $T$ and $T^{\prime}$ are times associated to the SUE ciphertext header and the SUE private key respectively. Thus, CDE.Decaps can derive a session key since the SUE ciphertext header consists of many CDE ciphertext header with labels in $\operatorname{TimeNodes}(\psi(T))$ and the SUE private key is equal to the CDE private key.

We now show that the output of SUE.UpdateCT is a valid ciphertext header. Recall that an SUE ciphertext header $\mathrm{CH}_{T}$ with time $T$ consists of $\mathrm{CDE}$ ciphertext headers that are associated with labels in TimeNodes $(\psi(T))$. Let $v_{T}$ be a node in $\mathcal{B} \mathcal{T}$ associated with time $T$ by pre-order traversal. If $v_{T}$ is an internal node, we have $v_{T+1}=\operatorname{LeftChild}\left(v_{T}\right)$ from Remark 5. Thus we have $\operatorname{TimeNodes}(\psi(T+1)) \backslash$ $\operatorname{TimeNodes}(\psi(T))=\left\{\operatorname{LeftChild}\left(v_{T}\right), \operatorname{RightChild}\left(v_{T}\right)\right\}$ since $\operatorname{RightSibling}\left(v_{T+1}\right)=\operatorname{RightChild}\left(v_{T}\right)$ by the definition of TimeNodes. If $v_{T}$ is a leaf node, $v_{T+1}=\operatorname{RightChild}\left(v_{T^{\prime}}\right)$ where $v_{T^{\prime}} \in \operatorname{Path}\left(v_{T}\right)$ is a node with the largest depth such that $\operatorname{LeftChild}\left(v_{T^{\prime}}\right) \in \operatorname{Path}\left(v_{T}\right)$ from $\operatorname{Remark} 5$. Thus we have $\operatorname{TimeNodes}(\psi(T)) \backslash$ TimeNodes $(\psi(T+1))=v_{T}$. Therefore, the correctness of SUE.UpdateCT is easily obtained if the output of CDE.DelegateCT is a valid CDE ciphertext header.

\subsection{Security Analysis}

To prove the TEC-CCA security of the above SUE scheme, we use the well-known partitioning method. To simplify the security proof, we use the meta-simulation technique of Lee [21]. In the meta-simulation technique, a simulator (meta-simulator) for the TEC-CCA security proof uses the previous simulator for 
the CPA security proof as a sub-simulator. Recall that the original SUE scheme of the above SUE scheme was proven to be selectively secure under the DBDH assumption by Lee [21]. If the meta-simulator use the previous simulator as a sub-simulator, then it can use the power of the sub-simulator to generate private keys and some elements of a challenge ciphertext header.

Theorem 3.2 ([21]). The original SUE scheme is selectively secure under chosen plaintext attacks if the DBDH assumption holds.

The simulator of this proof sets the element $g$ in the assumption $\left(g, g^{a}, g^{b}, g^{c}\right)$ as the generator of public parameters, implicitly sets $a b$ as the master key $\beta$, and sets $g^{c}$ in the assumption as the element $g^{t}$ in a challenge ciphertext header. Note that these three settings are essential for the correctness of the metasimulation. Now we can prove the TEC-CCA security of our SUE scheme as follows:

Theorem 3.3. The above SUE scheme is selectively secure under time extended challenge chosen ciphertext attacks if the DBDH assumption holds. That is, for any PPT adversary $\mathcal{A}$, we have that $\boldsymbol{A d v} v_{\mathcal{A}}^{S U E}(\lambda) \leq$ $\boldsymbol{A d v} v_{\mathcal{B}}^{D B D H}(\lambda)+\operatorname{negl}(\lambda)$.

Proof. Suppose there exists an adversary $\mathcal{A}$ that attacks the above SUE scheme with a non-negligible advantage. A simulator $\mathcal{B}$ that solves the $\mathrm{DBDH}$ assumption using $\mathcal{A}$ is given: a challenge tuple $D=$ $\left(\left(p, \mathbb{G}, \mathbb{G}_{T}, e\right), g, g^{a}, g^{b}, g^{c}\right)$ and $Z$ where $Z=Z_{0}=e(g, g)^{a b c}$ or $Z=Z_{1} \in \mathbb{G}_{T}$. Let $\mathcal{B}_{S U E}$ be a simulator in the security proof of Theorem 3.2 Then $\mathcal{B}$ that interacts with $\mathcal{A}$ is described as follows:

Init: $\mathcal{A}$ initially submits challenge time $T^{*} \cdot \mathcal{B}$ first runs $\mathcal{B}_{S U E}$ by giving $D$ and $Z$.

Setup: $\mathcal{B}$ submits $T^{*}$ to $\mathcal{B}_{S U E}$ and receives $P P_{S U E}=\left(\left(p, \mathbb{G}, \mathbb{G}_{T}, e\right), g, w, v, u,\left\{h_{i, j}\right\}, \Lambda=e(g, g)^{\beta}\right)$. Note that $\mathcal{B}_{S U E}$ implicitly sets $\beta=a b$. It chooses random exponents $u_{S}^{\prime}, h_{S}^{\prime} \in \mathbb{Z}_{p}$. It also chooses a random index $z$ for $H^{z}$ and calculates $\pi^{*}=H^{z}\left(g^{c}\right)$. It implicitly sets $\beta=a b$ and publishes public parameters $P P=$ $\left(P P_{S U E}, z, u_{S}=g^{a} g^{u_{S}^{\prime}}, h_{S}=\left(g^{a}\right)^{-\pi^{*}} g^{h_{S}^{\prime}}\right)$.

Query 1: $\mathcal{A}$ adaptively requests a polynomial number of private key queries and decryption queries. If $\mathcal{A}$ requests a private key query for time $T$ such that $T<T^{*}$, then $\mathcal{B}$ receives a private key $S K_{T}$ by requesting a private key query to $\mathcal{B}_{S U E}$ and responses $S K_{T}$ to $\mathcal{A}$. If $\mathcal{A}$ requests a decryption query for a ciphertext header $\mathrm{CH}_{T}$ with time $T$, then $\mathcal{B}$ handles this query as follows:

1. Let $\mathrm{CH}_{T}=\left(\mathrm{CH}^{(0)}, \ldots, \mathrm{CH}^{(d)}, C_{3}\right)$ and $C H^{(0)}=\left(C_{0}, C_{1},\left\{C_{i, 1}, C_{i, 2}\right\}\right)$. It first checks whether the ciphertext header $\mathrm{CH}_{T}$ is valid or not by running SUE.VerifyCT $\left(\mathrm{CH}_{T}, T, P P\right)$. If the ciphertext header is not valid, then it responds with $\perp$. Otherwise, the ciphertext header is well-formed such as $C_{0}=g^{t}$ for some unknown $t \in \mathbb{Z}_{p}$.

2. If $T<T^{*}$, then it receives a private key $S K_{T}$ by requesting a private key query to $\mathcal{B}_{S U E}$ and obtains a session key $E K$ by running SUE.Decaps $\left(\mathrm{CH}_{T}, S K_{T}, P P\right)$.

3. If $T \geq T^{*}$, then it calculates $\pi=H^{z}\left(C_{0}\right)$ and performs the following steps: If $\pi=\pi^{*}$, then it terminates the simulation with $\mathcal{A}$ and halts since it cannot response. If $\pi \neq \pi^{*}$, then it selects a random exponent $r^{\prime} \in \mathbb{Z}_{p}$ and computes the session key by implicitly setting $r_{3}=-\left(u_{S}^{\prime} \pi+h_{S}^{\prime}\right) /\left(\pi-\pi^{*}\right)+r^{\prime}$ as

$$
\begin{aligned}
E K & =e\left(C_{0},\left(g^{b}\right)^{-\frac{u_{S}^{\prime} \pi+h_{S}^{\prime}}{\pi-\pi^{*}}}\left(u_{S}^{\pi} h_{S}\right)^{r^{\prime}}\right) \cdot e\left(C_{3},\left(g^{b}\right)^{\frac{1}{\pi-\pi^{*}}} g^{-r^{\prime}}\right) \\
& =e\left(g^{t}, g^{a b}\left(u_{S}^{\pi} h_{S}\right)^{r_{3}}\right) \cdot e\left(\left(u_{S}^{\pi} h_{S}\right)^{t}, g^{-r_{3}}\right)=e\left(g^{a}, g^{b}\right)^{t} .
\end{aligned}
$$

4. It responds to the query with the session key $E K$. 
Challenge: To create the challenge ciphertext header and the session key for the challenge time $T^{*}, \mathcal{B}$ proceeds as follows:

1. It first requests a challenge query to $\mathcal{B}_{S U E}$ and receives $C H^{*}=\left(C H^{(0)}, C H^{(1)}, \ldots, C H^{(d)}\right)$ and $E K^{*}$. Recall that $\mathcal{B}_{S U E}$ sets the group element $C_{0}$ in $\mathrm{CH}^{*}$ as $\mathrm{g}^{c}$ in the challenge tuple.

2. It sets $C_{3}=\left(g^{c}\right)^{u_{S}^{\prime}} \pi^{*}+h_{S}^{\prime}$. Note that this component is valid since $\left(g^{c}\right)^{u_{S}^{\prime}} \pi^{*}+h_{S}^{\prime}=\left(\left(g^{a} g^{u_{S}^{\prime}}\right)^{\pi^{*}} \cdot\left(g^{a}\right)^{-\pi^{*}} g^{h_{S}^{\prime}}\right)^{c}=$ $\left(u_{S}^{\pi^{*}} h_{S}\right)^{c}$ for $\pi^{*}=H^{z}\left(C_{0}\right)=H^{z}\left(g^{c}\right)$.

3. It sets the challenge ciphertext header $C H_{T^{*}}^{*}=\left(C H^{(0)}, C H^{(1)}, \ldots, C H^{(d)}, C_{3}\right)$ and gives $C H_{T^{*}}^{*}$ and $E K^{*}$ to $\mathcal{A}$.

Query 2: This phase is handled as the same as the query 1 phase since $\mathcal{B}$ can fix the group element $C_{0}=g^{c}$ for the challenge ciphertext header at the setup phase in the selective model. Note that $\mathrm{R}\left(\mathrm{CH}_{T^{*}}^{*}, \mathrm{CH}_{T}\right)=$ True if $\mathrm{CH}_{T}$ is a valid SUE ciphertext header, $T^{*} \leq T$, and $C_{0}^{*}=C_{0}$ from the Remark 8 . By the restriction of the TEC-CCA security model, $\mathcal{A}$ can request the decryption of any ciphertext header $\mathrm{CH}_{T}$ such that $R\left(\mathrm{CH}_{T^{*}}^{*}, \mathrm{CH}_{T}\right)=\mathrm{False}$ and these ciphertext headers are correctly handled in the query 1 phase since $\pi \neq \pi^{*}$.

Guess: $\mathcal{A}$ outputs a guess $\mu^{\prime}$. $\mathcal{B}$ also outputs $\mu^{\prime}$.

To finish the proof, we show that the meta-simulator $\mathcal{B}$ correctly handles the queries of $\mathcal{A}$. The public parameters $P P$ is correct since $P P_{S U E}$ is correct and $u_{S}, h_{S}$ are properly randomized. The private key is also correct since it is generated by $\mathcal{B}_{S U E}$. Now, we will show that the decryption query is correctly handled. From the correctness of the SUE.VerifyCT algorithm, we confirm that the ciphertext header $\mathrm{CH}_{T}$ is associated with claimed time $T$. The decryption only fails when $T \geq T^{*}$ and $\pi=\pi^{*}$ where $\pi=H^{z}\left(C_{0}\right)$ and $\pi^{*}=H^{z}\left(g^{c}\right)$. The probability of this collision event is negligible since $H^{z}$ is a collision resistant function and $C_{0} \neq g^{c}$ from the restriction of the security model. Finally, the challenge ciphertext is also correct since $C_{3}$ can be easily calculated. This completes our proof.

Corollary 3.4. The above SUE scheme is fully secure under time extended challenge chosen ciphertext attacks if the DBDH assumption holds and $T_{\max }$ is a polynomial value. That is, for any PPT adversary $\mathcal{A}$, we have that $\boldsymbol{A d v} v_{\mathcal{A}}^{S U E}(\lambda) \leq T_{\max } \cdot \boldsymbol{A d v _ { \mathcal { B } } ^ { D B D H }}(\lambda)+\operatorname{negl}(\lambda)$.

\subsection{Discussions}

Efficiency Analysis. The proposed SUE scheme composed of $O\left(\log T_{\max }\right)$ group elements in the public parameters, a private key, and a ciphertext header since it utilizes the CDE scheme proposed by Lee [21]. Note that our SUE scheme additionally includes two group elements in the public parameters and one group element in a ciphertext header to provide the TEC-CCA security. Unlike the existing CPA secure SUE scheme, our TEC-CCA secure SUE scheme should check the validity of the ciphertext header in the decapsulation algorithm. If the SUE decapsulation algorithm checks the validity of CDE ciphertext headers by running the the CDE verification algorithm in a simple manner, then it requires $O\left(\log ^{2} T_{\max }\right)$ pairing operations since the $\mathrm{CDE}$ verification algorithm requires $O\left(\log T_{\max }\right)$ pairing operations and one SUE ciphertext header consists of $O\left(\log T_{\max }\right)$ CDE ciphertext headers. However, since there are duplicate values in CDE ciphertext headers, it is possible to reduce the pairing operation from $O\left(\log ^{2} T_{\max }\right)$ times to $O\left(\log T_{\max }\right)$ times during verification. See below in this section for this improvement.

Reducing Public Parameters. Since our SUE scheme uses the CDE scheme of Lee [21] secure under the $\mathrm{DBDH}$ assumption, the public parameter is composed of $O\left(\log T_{\max }\right)$ group elements. In order to reduce the size of the public parameter of the SUE scheme, another efficient CDE scheme having a short public 
parameter proposed by Lee [21] can be used. The public parameter of a TEC-CCA secure SUE scheme constructed in this way has $O(1)$ group elements instead of $O\left(\log T_{\max }\right)$ group elements, and the private key and the ciphertext are all kept in the same number of group elements as the previous SUE scheme. However, the security of this SUE scheme with short public parameters is only proven to be TEC-CCA secure under the $q$-type assumption instead of the standard DBDH assumption.

Time-Interval SUE. By combining two CPA secure SUE schemes, a CPA secure time-interval SUE (TISUE) scheme can be constructed as presented by Lee [21]. In TI-SUE, a ciphertext header is associated with a time range specified by two times $T_{L}$ and $T_{R}$ and a private key is associated with time $T^{\prime}$. If $T_{L} \leq T^{\prime} \leq T_{R}$, then the ciphertext with $T_{L}$ and $T_{R}$ can be decrypted by a private key with $T^{\prime}$. By following the design principle of Lee, we can also build a TEC-CCA secure TI-SUE scheme by combining two TEC-CCA secure SUE schemes. That is, the master key is simply shared between two SUE schemes to prevent collusion attacks, the ciphertext header of one SUE scheme is used for future-time SUE, and the ciphertext header of another SUE scheme is used for past-time SUE. This TI-SUE scheme also can be proven to be secure under the DBDH assumption. Note that we can also reduce the size of public parameters if the CDE scheme with short public parameters is used.

Improving Ciphertext Verification. The ciphertext header of the proposed SUE scheme consists of maximum $\log T_{\max } \mathrm{CDE}$ ciphertext headers and the verification of one CDE ciphertext header requires up to $2 \log T_{\max }+3$ pairing operations. Therefore, if the validity of the SUE ciphertext header is checked by a simple method, at most $2 \log ^{2} T_{\max }+3 \log T_{\max }+2$ pairing operations are required. As described above, one method of reducing the number of pairing operations in ciphertext verification is to omit the verification of duplicate elements. In other words, since the CDE ciphertext headers contained in one SUE ciphertext header share random exponents, the ciphertext elements associated with these shared random exponents can be performed only once at the time of $\mathrm{CH}^{(0)} \mathrm{CDE}$ ciphertext header verification, and can be omitted at a later time. In this case, $2 \log T_{\max }+3$ pairing operations are required for the verification of the $C H^{(0)} \mathrm{CDE}$ ciphertext header and the verification of the $\mathrm{CH}^{(i)} \mathrm{CDE}$ ciphertext header requires 4 pairing operations. Therefore, at most $6 \log T_{\max }+5$ pairing operations are required for the SUE ciphertext header verification.

Fast Verification by Batching. Another way to improve the performance of SUE ciphertext header verification is to use batch verification. Batch verification is a way of verifying the validity of all signatures by processing multiple signatures at once, rather than verifying individual signatures one at a time when multiple signatures are given [3,8]. In the SUE scheme, since one SUE ciphertext header consists of multiple CDE ciphertext headers, the total number of pairing operations can be reduced by performing batch verification using the small exponent test method on the CDE ciphertext headers after removing redundant elements. However, to properly use batch verification, we need to perform additional group membership tests to make sure that the group elements of ciphertext headers belong to the group. If batch verification is performed to one SUE ciphertext header, approximately $2 \log T_{\max }+8$ pairing operations and $5 \log T_{\max }$ group membership test operations are required. At this time, since the group membership test in a bilinear group is almost the same as the exponentiation operation, batch verification is effective only for the bilinear group in which the group exponentiation operation is faster than the pairing operation. Typically, in the case of the MNT curve, which is a Type 3 pairing, it is possible to perform more efficient ciphertext verification by using batch verification.

Lazy Ciphertext Updating. The ciphertext update algorithm of the proposed SUE scheme is very efficient because it performs only ciphertext delegation without performing ciphertext re-randomization. That is, in order to update one ciphertext of time $T$ to a ciphertext of time $T+1$, only a maximum of 3 exponentiation operations need be performed. However, when a large number of ciphertexts are stored in a repository and 
a server needs to update the ciphertexts every time, there is a problem that the total amount of computation for updating the ciphertexts is considerably large. One way to solve this problem is to not update the ciphertexts every time, but the server updates the ciphertext of past time $T_{1}$ to a ciphertext of current time $T_{2}$ when the ciphertext access occurs. In addition, when updating the ciphertext from time $T_{1}$ to time $T_{2}$, it is possible to update the ciphertext more efficiently by directly obtaining delegated ciphertexts from the set of TimeNode $\left(T_{2}\right)$ and TimeNode $\left(T_{1}\right)$ instead of performing the ciphertext updating algorithm for $T_{2}-T_{1}$ times.

\section{Revocable-Storage Attribute-Based Encryption}

In this section, we define the syntax and the TEC-CCA security of RS-ABE. We also propose an RS-ABE scheme and prove its TEC-CCA security.

\subsection{Definitions}

Revocable-Storage $\mathrm{ABE}$ (RS-ABE) is $\mathrm{ABE}$ that supports user revocation and ciphertext updating. The concept of RS-ABE was introduced by Sahai et al. [30] to handle the access control problem on ciphertexts in cloud storage. In this paper, we follow the RS-ABE syntax of Lee [21]. In RS-ABE with ciphertext-policy, a user's private key is associated with a set of attributes $S$ and an index $u$ and a ciphertext is associated with an access structure $\mathbb{A}$ and time $T$. A center periodically broadcast an update key that excludes a set of revoked users $R$ on time $T$. If a user is not revoked $(u \notin R)$ and the set of attributes satisfies the access structure $(S \in \mathbb{A})$, then the user with a private key and an update key can decrypt the ciphertext. The syntax of $\mathrm{RS}-\mathrm{ABE}$ is given as follows:

Definition 4.1 (Revocable-Storage Attribute-Based Encryption). A revocable-storage (ciphertext-policy) attribute-based encryption (RS-ABE) scheme for the universe of attributes $\mathcal{U}$ consists of seven PPT algorithms, Setup, GenKey, UpdateKey, DeriveKey, Encaps, UpdateCT, and Decaps, which are defined as follows:

$\operatorname{Setup}\left(1^{\lambda}, T_{\max }, N_{\max }\right)$. The setup algorithm takes as input a security parameter $1^{\lambda}$, the maximum time $T_{\max }$, and the maximum number of users $N_{\max }$, and it outputs a master key $M K$ and public parameters $P P$.

$\operatorname{GenKey}(S, u, M K, P P)$. The key generation algorithm takes as input a set of attributes $S \subseteq \mathcal{U}$, a user index $u \in \mathcal{N}$, the master key $M K$, and the public parameters $P P$. It outputs a private key $S K_{S, u}$.

$\operatorname{UpdateKey}(T, R, M K, P P)$. The key update algorithm takes as input time $T \leq T_{\max }$, a set of revoked users $R \subseteq \mathcal{N}$, the master key $M K$, and the public parameters $P P$. It outputs an update key $U K_{T, R}$.

DeriveKey $\left(S K_{S, u}, U K_{T, R}, P P\right)$. The decryption key derivation algorithm takes as input a private key $S K_{S, u}$, an update key $U K_{T, R}$, and the public parameters $P P$. It outputs a decryption key $D K_{S, T}$ or the distinguished symbol $\perp$.

$\operatorname{Encaps}(\mathbb{A}, T, P P)$. The encapsulation algorithm takes as input an access structure $\mathbb{A}$, time $T \leq T_{\max }$, and the public parameters $P P$. It outputs a ciphertext header $C H_{\mathbb{A}, T}$ and a session key $E K$.

UpdateCT $\left(\mathrm{CH}_{\mathbb{A}, T}, T+1, P P\right)$. The ciphertext update algorithm takes as input a ciphertext header $C H_{\mathbb{A}, T}$, new time $T+1$ such that $T+1 \leq T_{\max }$, and the public parameters $P P$. It outputs an updated ciphertext header $C H_{\mathbb{A}, T+1}$. 
Decaps $\left(C H_{\mathbb{A}, T}, D K_{S, T^{\prime}}, P P\right)$. The decapsulation algorithm takes as input a ciphertext header $C H_{\mathbb{A}, T}$, a decryption key $D K_{S, T}$, and the public parameters $P P$. It outputs a session key $E K$ or the distinguished symbol $\perp$.

The correctness of RS-ABE is defined as follows: For all $P P, M K$ generated by Setup, all $S$ and $u$, any $S K_{S, u}$ generated by GenKey, all $\mathbb{A}, T$, any $C H_{\mathbb{A}, T}, E K$ generated by Encaps or UpdateCT, all $T^{\prime}$ and $R$, any $U K_{T^{\prime}, R}$ generated by UpdateKey, it is required that:

- If $u \notin R$, then DeriveKey $\left(S K_{S, u}, U K_{T^{\prime}, R}, P P\right)=D K_{S, T^{\prime}}$.

- If $u \in R$, then DeriveKey $\left(S K_{S, u}, U K_{T^{\prime}, R}, P P\right)=\perp$ with all but negligible probability.

- If $(S \in \mathbb{A}) \wedge\left(T \leq T^{\prime}\right)$, then Decaps $\left(C H_{\mathbb{A}, T}, D K_{S, T^{\prime}}, P P\right)=E K$.

- If $(S \notin \mathbb{A}) \vee\left(T^{\prime}<T\right)$, then Decaps $\left(\mathrm{CH}_{\mathbb{A}, T}, D K_{S, T^{\prime}}, P P\right)=\perp$ with all but negligible probability.

Additionally, it requires that the updated ciphertext header of UpdateCT is a valid ciphertext header under the new time.

Remark 9. The previous definition of CPA secure RS-ABE additionally contains the RandCT algorithm that randomizes a ciphertext header [21, 22, 30]. However, our definition of RS-ABE with TEC-CCA security omits the RandCT algorithm because of the reason in Remark 11. Thus, the output of the UpdateCT algorithm is a just valid ciphertext header, and the randomness of an updated ciphertext header may be correlated to that of the original ciphertext header. Because of this correlation, if a ciphertext header is updated from an original one by using UpdateCT, then the original one should be deleted.

Remark 10. We define RS-ABE as a key encapsulation mechanism (KEM) version, in which the Encaps algorithm derives a session key, instead of a full encryption version. Note that if a KEM scheme is combined with a symmetric key encryption scheme, then a full encryption scheme can be easily derive by using the hybrid encryption technique.

The CPA security of RS-ABE was introduced by Sahai et al. [30] and refined by Lee [21] to consider the decryption key exposure attack. We define the TEC-CCA security of RS-ABE by modifying their CPAsecurity model. Similar to the CCA security of SUE, RS-ABE also cannot achieve the traditional CCA2 security since the ciphertexts of RS-ABE can be updated by anyone. Thus, we also relax the definition of CCA2 security by restricting that an adversary cannot request a decryption query on a ciphertext that is updated from the challenge ciphertext given to the adversary. In this paper, we define selective time extended challenge (TEC) CCA security of RS-ABE where the adversary should submit a challenge access structure and a challenge time before he receives public parameters. The TEC-CCA security of RS-ABE is given as follows:

Definition 4.2 (Selective TEC-CCA Security). The selective security of RS-ABE is defined in terms of the indistinguishability under time extended challenge chosen ciphertext attacks (IND-TEC-CCA). The security game is defined as the following experiment between a challenger $\mathcal{C}$ and a PPT adversary $\mathcal{A}$ :

Init: $\mathcal{A}$ first submits a challenge access structure $\mathbb{A}^{*}$ and challenge time $T^{*}$.

Setup: $\mathcal{C}$ generates a master key $M K$ and public parameters $P P$ by calling the setup algorithm, and then it gives $P P$ to $\mathcal{A}$.

Query 1: $\mathcal{A}$ may adaptively request a polynomial number of private key, update key, decryption key, and decryption queries. $\mathcal{C}$ handles the queries as follows: 
- If this is a private key query for a set of attributes $S$ and a user index $u$, then it creates a private key $S K_{S, u}$ by calling the key generation algorithm and gives $S K_{S, u}$ to $\mathcal{A}$. Note that $\mathcal{A}$ is allowed to query only one private key for each user $u$.

- If this is an update key query for time $T$ and a set of revoked users $R$, then it creates an update key $U K_{T, R}$ by calling the key update algorithm and gives $U K_{T, R}$ to $\mathcal{A}$. Note that $\mathcal{A}$ is allowed to query only one update key for each time $T$.

- If this is a decryption key query for a set of attributes $S$ and time $T$, then it creates a decryption key $D K_{S, T}$ by calling the decryption key derivation algorithm and gives $D K_{S, T}$ to $\mathcal{A}$.

- If this is a decryption query for a ciphertext header $C H_{\mathbb{A}, T}$, then it computes the decapsulated session key $E K$ by calling the decryption algorithm and gives $E K$ to $\mathcal{A}$.

We require the following restrictions on the queries of $\mathcal{A}$ :

1. If an update key for $T$ and $R$ was queried, then $R \subseteq R_{j}$ for all update key queries on $T_{j}$ and $R_{j}$ such that $T<T_{j}$.

2. If a private key for $S$ and $u$ such that $S \in \mathbb{A}^{*}$ was queried, then an update key for $T_{j}$ and $R_{j}$ such that $u \in R_{j}$ and $T_{j} \leq T^{*}$ should be queried to revoke this user index $u$.

3. If a decryption key for $S$ and $T$ was queried, then it is required that $S \notin \mathbb{A}^{*}$ or $T<T^{*}$.

Challenge: $\mathcal{C}$ creates a ciphertext header $C H_{\mathbb{A}^{*}, T^{*}}^{*}$ and a session key $E K^{*}$ by calling the encryption algorithm under the challenge access structure $\mathbb{A}^{*}$ and the challenge time $T^{*}$. It then flips a random bit $\mu \in\{0,1\}$. If $\mu=0$ it sets $E K_{0}^{*}=E K^{*}$, otherwise it sets $E K_{1}^{*}$ to a random session key. It gives $C H_{\mathbb{A}^{*}, T^{*}}^{*}$ and $E K_{\mu}^{*}$ to $\mathcal{A}$. Note that $\mathcal{A}$ can obtain updated ciphertext headers that are derived from the challenge ciphertext header since the ciphertext update algorithm can be performed by anyone.

Query 2: $\mathcal{A}$ continues to request private key, update key, decryption key, and decryption queries. $\mathcal{C}$ handles the queries as the same as before. In addition to the restrictions in query $1 \mathrm{step}$, we require the following additional restriction on the queries of $\mathcal{A}$ :

4. If a decryption for a ciphertext header $C H_{\mathbb{A}^{*}, T}$ was queried, then it is required that $T<T^{*}$ or $C H_{\mathbb{A}^{*}, T}$ is not updated from $C H_{\mathbb{A}^{*}, T^{*}}^{*}$ for $T \geq T^{*}$.

Guess: Finally $\mathcal{A}$ outputs a bit $\mu^{\prime}$.

The advantage of $\mathcal{A}$ is defined as $\operatorname{Adv}_{\mathcal{A}}^{R S-A B E}(\lambda)=\left|\operatorname{Pr}\left[\mu=\mu^{\prime}\right]-\frac{1}{2}\right|$ where the probability is taken over all the randomness of the game. An RS-ABE scheme is secure in the selective model under time extended challenge chosen ciphertext attacks if for all PPT adversaries $\mathcal{A}$, the advantage of $\mathcal{A}$ in the above game is negligible in the security parameter $\lambda$.

Remark 11. The adversary of the above TEC-CCA security model cannot request a decryption on an updated ciphertext header that is updated from the challenge ciphertext header. Thus, there should be an efficiently computable relation $R(-,-)$ that checks whether a ciphertext header is derived from another one or not. If an RS-ABE scheme supports perfect ciphertext re-randomization, then the security of this scheme cannot be proven in the above model since there is no efficiently computable relation $R(-,-)$.

Remark 12. Selective TEC-CCA security can be weakened to selective revocation list TEC-CCA security, in which an adversary should additionally submits a set of revoked users on the challenge time. Selective revocation list CPA security was introduced by Boldyreva et al. [4] to prove the security of their RS-ABE scheme and this is employed in RS-ABE by Lee [21]. Note that selective TEC-CCA security is stronger than selective revocation list TEC-CCA security. 


\subsection{Subset Cover Framework}

The subset cover (SC) framework, introduced by Naor, Naor, and Lotspiech [25], is a general methodology to construct an efficient revocation system. The complete subtree (CS) scheme is one instance of the SC framework. We follow the definition of CS in [22]. The CS scheme is given as follows:

CS.Setup $\left(N_{\max }\right)$ : This algorithm takes as input the maximum number of users $N_{\max }$. Let $N_{\max }=2^{d}$ for simplicity. It first sets a full binary tree $\mathcal{B} \mathcal{T}$ of depth $d$. Each user is assigned to a different leaf node in $\mathcal{B T}$. The collection $\mathcal{S}$ is defined as $\left\{S_{i}: v_{i} \in \mathcal{B T}\right\}$. Recall that $S_{i}$ is the set of all the leaves in a subtree $\mathcal{T}_{i}$. It outputs $\mathcal{B} \mathcal{T}$.

$\operatorname{CS.Assign}(\mathcal{B T}, u)$ : This algorithm takes as input the tree $\mathcal{B T}$ and a user $u \in \mathcal{N}$. Let $v_{u}$ be the leaf node of $\mathcal{B T}$ that is assigned to the user $u$. Let $\left(v_{j_{0}}, v_{j_{1}}, \ldots, v_{j_{d}}\right)$ be the path from the root node $v_{j_{0}}=v_{0}$ to the leaf node $v_{j_{n}}=v_{u}$. It sets $P V_{u}=\left\{S_{j_{0}}, \ldots, S_{j_{d}}\right\}$ and outputs the private set $P V_{u}$.

CS.Cover $(\mathcal{B T}, R)$ : This algorithm takes as input the tree $\mathcal{B T}$ and a revoked set $R$ of users. It first computes the Steiner tree $S T(R)$. Let $\mathcal{T}_{i_{1}}, \ldots \mathcal{T}_{i_{m}}$ be all the subtrees of $\mathcal{B} \mathcal{T}$ that hang off $S T(R)$, that is all subtrees whose roots $v_{i_{1}}, \ldots v_{i_{m}}$ are not in $S T(R)$ but adjacent to nodes of outdegree 1 in $S T(R)$. It outputs a covering set $C V_{R}=\left\{S_{i_{1}}, \ldots, S_{i_{m}}\right\}$.

CS.Match $\left(C V_{R}, P V_{u}\right)$ : This algorithm takes input as a covering set $C V_{R}=\left\{S_{i_{1}}, \ldots, S_{i_{m}}\right\}$ and a private set $P V_{u}=\left\{S_{j_{0}}, \ldots, S_{j_{d}}\right\}$. It finds a subset $S_{k}$ with $S_{k} \in C V_{R}$ and $S_{k} \in P V_{u}$. If there is such a subset, it outputs $\left(S_{k}, S_{k}\right)$. Otherwise, it outputs $\perp$.

Lemma 4.1 ( [25]). Let $N_{\max }$ be the number of leaf nodes in a full binary tree and $r$ be the size of a revoked set. In the CS scheme, the size of a private set is $\log N_{\text {max }}$ and the size of a covering set is at most $r \log \left(N_{\max } / r\right)$.

\subsection{Construction}

Before we construct an RS-ABE scheme, we present a CCA secure CP-ABE scheme from the CPA secure $\mathrm{CP}-\mathrm{ABE}$ scheme of Rouselakis and Waters [29]. To convert the CPA secure CP-ABE scheme to a CCA secure one, we follow the transformation of Canetti et al. [9]. To improve the efficiency, we actually employ the direct conversion method of Boyen et al. [7]. The KEM version of the CP-ABE scheme is given as follows:

CP-ABE.Setup $(G D S)$ : This algorithm takes as input a group description string GDS. It chooses random elements $w_{A}, v_{A}, u_{A}, h_{A}, u_{B}, h_{B} \in \mathbb{G}$, and a random exponent $\gamma \in \mathbb{Z}_{p}$. It also chooses a random index $z$ for a hash function $H^{z} \in \mathcal{H}$. It outputs a master key $M K=\gamma$ and public parameters $P P=\left(\left(p, \mathbb{G}, \mathbb{G}_{T}, e\right), g, w_{A}, v_{A}, u_{A}, h_{A}, z, u_{B}, h_{B}, \Lambda=e(g, g)^{\gamma}\right)$.

CP-ABE.GenKey $(S, M K, P P)$ : Let $S=\left\{A_{1}, A_{2}, \ldots, A_{k}\right\}$ be a set of attributes. It chooses random exponents $r, r_{1}, \ldots, r_{k} \in \mathbb{Z}_{p}$ and outputs a private key $S K_{S}=\left(K_{0}=g^{\gamma} w_{A}^{r}, K_{1}=g^{-r},\left\{K_{i, 2}=v_{A}^{r}\left(u_{A}^{A_{i}} h_{A}\right)^{r_{i}}, K_{i, 3}=\right.\right.$ $\left.\left.g^{-r_{i}}\right\}_{i=1}^{k}\right)$.

CP-ABE.RandKey $\left(S K_{S}, \delta, P P\right)$ : Let $S K_{S}=\left(K_{0}, K_{1},\left\{K_{i, 2}, K_{i, 3}\right\}\right)$ for $S=\left\{A_{1}, A_{2}, \ldots, A_{k}\right\}$. It chooses random exponents $r^{\prime}, r_{1}^{\prime}, \ldots, r_{k}^{\prime} \in \mathbb{Z}_{p}$ and outputs a re-randomized private key $S K_{S}=\left(K_{0}^{\prime}=K_{0} \cdot g{ }^{\delta} w_{A}^{r^{\prime}}, K_{1}^{\prime}=\right.$ $\left.K_{1} \cdot g^{-r^{\prime}},\left\{K_{i, 2}^{\prime}=K_{i, 2} \cdot v_{A}^{r^{\prime}}\left(u_{A}^{A_{i}} h_{A}\right)^{r_{i}^{\prime}}, K_{i, 3}^{\prime}=K_{i, 3} \cdot g^{-r_{i}^{\prime}}\right\}_{i=1}^{k}\right)$. 
CP-ABE.Encaps $(\mathbb{A}, t, P P)$ : Let $\mathbb{A}=(M, \rho)$ be an LSSS access structure where $M$ is an $l \times n$ matrix and $\rho$ is a map from each row $M_{j}$ of $M$ to an attribute $\rho(j)$. It first sets a random vector $\vec{v}=\left(t, v_{2}, \ldots, v_{n}\right)$ by selecting random exponents $v_{2}, \ldots, v_{n} \in \mathbb{Z}_{p}$. It selects random exponents $s_{1}, \ldots, s_{l} \in \mathbb{Z}_{p}$ and computes $C_{0}=g^{t},\left\{C_{j, 1}=w_{A}^{M_{j} \cdot \vec{v}} v_{A}^{s_{j}}, C_{j, 2}=g^{s_{j}}, C_{j, 3}=\left(u_{A}^{\rho(j)} h_{A}\right)^{s_{j}}\right\}_{1 \leq j \leq l}$. Next, it calculates $\pi=H^{z}\left(C_{0}, C_{1,1}, \ldots, C_{l, 3}\right)$ and sets $C_{3}=\left(u_{B}^{\pi} h_{B}\right)^{t}$. It outputs a ciphertext header $C H_{\mathbb{A}}=\left(C_{0},\left\{C_{j, 1}, C_{j, 2}, C_{j, 3}\right\}_{j=1}^{l}, C_{3}\right)$ and a session key $E K=\Lambda^{t}$.

CP-ABE.VerifyCT $\left(C_{\mathbb{A}}, P P\right)$ : Let $C H_{\mathbb{A}}=\left(C_{0},\left\{C_{j, 1}, C_{j, 2}, C_{j, 3}\right\}, C_{3}\right)$. It first computes $\pi=H^{z}\left(C_{0}, C_{1,1}, \ldots\right.$, $\left.C_{l, 3}\right)$ and checks $e\left(C_{3}, g\right) \stackrel{?}{=} e\left(C_{0}, u_{B}^{\pi} h_{B}\right)$. It outputs 1 if the check passes. Otherwise, it outputs 0 .

CP-ABE.Decaps $\left(\mathrm{CH}_{\mathbb{A}}, S K_{S}, P P\right)$ : Let $C H_{\mathbb{A}}=\left(C_{0},\left\{C_{j, 1}, C_{j, 2}, C_{j, 3}\right\}, C_{3}\right)$ and $S K_{S}=\left(K_{0}, K_{1},\left\{K_{j, 2}, K_{j, 3}\right\}\right)$. It first checks $1 \stackrel{?}{=} \mathbf{C P}$-ABE.VerifyCT $\left(\mathrm{CH}_{\mathbb{A}}, P P\right)$. If the checking fails, then it outputs $\perp$. If $S \in \mathbb{A}$, then it computes constants $\omega_{j} \in \mathbb{Z}_{p}$ such that $\sum_{\rho(j) \in S} \omega_{j} M_{j}=(1,0, \ldots, 0)$ and outputs a session key as $E K=e\left(C_{0}, K_{0}\right) \cdot \prod_{\rho(j) \in S}\left(e\left(C_{j, 1}, K_{1}\right) \cdot e\left(C_{j, 2}, K_{j, 2}\right) \cdot e\left(C_{j, 3}, K_{j, 3}\right)\right)^{\omega_{j}}$. Otherwise, it outputs $\perp$.

Remark 13. Compared to the CPA secure CP-ABE scheme [29], the above CP-ABE scheme additionally contains a hash function index $z$ and two group elements $u_{B}, h_{B}$ in public parameters, and a group element $C_{3}$ in a ciphertext header for integrity. We also added the RandKey algorithm to randomize a private key and the VerifyCT algorithm to check the validity of ciphertext headers. These two additional algorithms are used in the construction of an RS-ABE scheme.

To construct an RS-ABE scheme, we follow the design principle of Lee et al. [22]. Our RS-ABE scheme that uses the above CP-ABE scheme, our SUE scheme, and the CS scheme is described as follows:

RS-ABE.Setup $\left(1^{\lambda}, T_{\max }, N_{\max }\right)$ : It first generates bilinear groups $\mathbb{G}, \mathbb{G}_{T}$ of prime order $p$. Let $g$ be the generator of $\mathbb{G}$. It sets $G D S=\left(\left(p, \mathbb{G}, \mathbb{G}_{T}, e\right), g\right)$. It obtains $M K_{A B E}, P P_{A B E}$ and $M K_{S U E}, P P_{S U E}$ by running CP-ABE.Setup $(G D S)$ and SUE.Setup $\left(G D S, T_{\max }\right)$ respectively. It also obtains $\mathcal{B} \mathcal{T}$ by running $\operatorname{CS.Setup}\left(N_{\max }\right)$ and assigns a random exponent $\gamma_{i} \in \mathbb{Z}_{p}$ to each node $v_{i}$ in $\mathcal{B} \mathcal{T}$. It selects a random exponent $\alpha \in \mathbb{Z}_{p}$, and outputs a master key $M K=\left(M K_{A B E}, M K_{S U E}, \alpha, \mathcal{B} \mathcal{T}\right)$ and public parameters $P P=\left(P P_{A B E}, P P_{S U E}, \Omega=e(g, g)^{\alpha}\right)$.

RS-ABE.GenKey $(S, u, M K, P P)$ : Let $M K=\left(M K_{A B E}, M K_{S U E}, \alpha, \mathcal{B T}\right)$. It first assigns the index $u$ to a random leaf node $v_{u} \in \mathcal{B T}$. It obtains a private set $P V_{u}=\left\{S_{j_{0}}^{\prime}, \ldots, S_{j_{d}}^{\prime}\right\}$ by running $\operatorname{CS} \operatorname{Assign}(\mathcal{B} \mathcal{T}, u)$ and retrieves $\left\{\gamma_{j_{0}}, \ldots, \gamma_{j_{d}}\right\}$ from $\mathcal{B T}$ where $S_{j_{k}}^{\prime}$ is associated with a node $v_{j_{k}}$ and $\gamma_{j_{k}}$ is assigned to the node $v_{j_{k}}$. For $0 \leq k \leq d$, it sets $M K_{A B E}^{\prime}=\gamma_{j_{k}}$ and obtains $S K_{A B E, k}$ by running CP-ABE.GenKey $\left(S, M K_{A B E}^{\prime}\right.$, $\left.P P_{A B E}\right)$. It outputs a private key $S K_{S, u}=\left(P V_{u}, S K_{A B E, 0}, \ldots, S K_{A B E, d}\right)$.

RS-ABE.UpdateKey $(T, R, M K, P P)$ : Let $M K=\left(M K_{A B E}, M K_{S U E}, \alpha, \mathcal{B T}\right)$. It obtains a covering set $C V_{R}=$ $\left\{S_{i_{1}}^{\prime}, \ldots, S_{i_{m}}^{\prime}\right\}$ by running $\operatorname{CS}$. $\operatorname{Cover}(\mathcal{B} \mathcal{T}, R)$ and retrieves $\left\{\gamma_{i_{1}}, \ldots, \gamma_{i_{m}}\right\}$ from $\mathcal{B} \mathcal{T}$ where $S_{i_{k}}^{\prime}$ is associated with a node $v_{i_{k}}$ and $\gamma_{i_{k}}$ is assigned to the node $v_{i_{k}}$. For $1 \leq k \leq m$, it sets $M K_{S U E}^{\prime}=\alpha-\gamma_{i_{k}}$ and obtains $S K_{S U E, k}$ by running SUE.GenKey $\left(T, M K_{S U E}^{\prime}, P P_{S U E}\right)$. It outputs an update key $U K_{T, R}=$ $\left(C V_{R}, S K_{S U E, 1}, \ldots, S K_{S U E, m}\right)$.

RS-ABE.DeriveKey $\left(S K_{S, u}, U K_{T^{\prime}, R}, P P\right):$ Let $S K_{S, u}=\left(P V_{u}, S K_{A B E, 0}, \ldots, S K_{A B E, d}\right)$ and $U K_{T^{\prime}, R}=\left(C V_{R}, S K_{S U E, 1}\right.$, $\left.\ldots, S K_{S U E, m}\right)$. If $u \notin R$, then it obtains $\left(S_{i}, S_{j}\right)$ by running $\mathbf{C S . M a t c h}\left(C V_{R}, P V_{u}\right)$. Otherwise, it outputs $\perp$. It selects a random exponent $\delta \in \mathbb{Z}_{p}$ and obtains $S K_{A B E}$ by running CP-ABE. $\operatorname{RandKey}\left(\delta, S K_{A B E, j}\right.$, $\left.P P_{A B E}\right)$. It also obtains $S K_{S U E}$ by running $\mathbf{S U E} \cdot \operatorname{RandKey}\left(-\delta, S K_{S U E, i}, P P_{S U E}\right)$. It outputs a decryption key $D K_{S, T^{\prime}}=\left(S K_{A B E}, S K_{S U E}\right)$. 
RS-ABE.Encaps $(\mathbb{A}, T, P P)$ : It selects a random exponent $t \in \mathbb{Z}_{p}$ and obtains $C H_{A B E}$ and $C H_{S U E}$ by running CP-ABE.Encaps $\left(\mathbb{A}, t, P P_{A B E}\right)$ and $\mathbf{S U E . E n c a p s}\left(T, t, P P_{S U E}\right)$ respectively. Note that it ignores two partial session keys that are returned by CP-ABE.Encaps and SUE.Encaps. It outputs a ciphertext header $C H_{\mathbb{A}, T}=\left(C H_{A B E}, C H_{S U E}\right)$ and a session key $E K=\Omega^{t}$.

RS-ABE.UpdateCT $\left(\mathrm{CH}_{\mathbb{A}, T}, T+1, P P\right)$ : Let $\mathrm{CH}_{\mathbb{A}, T}=\left(\mathrm{CH}_{A B E}, \mathrm{CH}_{S U E}\right)$. It first obtains $\mathrm{CH}_{S U E}^{\prime}$ by running SUE.UpdateCT $\left(\mathrm{CH}_{S U E}, T+1, P P_{S U E}\right)$. It outputs an updated ciphertext header $C H_{\mathbb{A}, T+1}=$ $\left(\mathrm{CH}_{A B E}, \mathrm{CH}_{S U E}^{\prime}\right)$.

RS-ABE.VerifyCT $\left(\mathrm{CH}_{\mathbb{A}, T}, P P\right)$ : Let $\mathrm{CH}_{\mathbb{A}, T}=\left(\mathrm{CH}_{A B E}, \mathrm{CH}_{S U E}\right), \mathrm{CH}_{A B E}=\left(\mathrm{C}_{0}, \ldots\right)$, and $C H_{S U E}=\left(C H^{(0)}, \ldots\right)$ where $C H^{(0)}=\left(C_{0}^{\prime}, \ldots\right)$. It first checks $C_{0} \stackrel{?}{=} C_{0}^{\prime}$. It also checks that $1 \stackrel{?}{=}$ SUE.VerifyCT $\left(C H_{S U E}, T, P P_{S U E}\right)$ and $1 \stackrel{?}{=} \mathbf{C P}$-ABE.VerifyCT $\left(\mathrm{CH}_{A B E}, P P_{A B E}\right)$. If all checking pass, it outputs 1 . Otherwise, it outputs $\perp$.

RS-ABE.Decaps $\left(\mathrm{CH}_{\mathbb{A}, T}, D K_{S, T^{\prime}}, P P\right)$ : Let $C H_{\mathbb{A}, T}=\left(\mathrm{CH}_{A B E}, C H_{S U E}\right)$ and $D K_{S, T^{\prime}}=\left(S K_{A B E}, S K_{S U E}\right)$. Let $C H_{A B E}=\left(C_{0}, \ldots\right)$ and $C H_{S U E}=\left(C H^{(0)}, \ldots\right)$ where $C H^{(0)}=\left(C_{0}^{\prime}, \ldots\right)$. It first checks $C_{0} \stackrel{?}{=} C_{0}^{\prime}$. If the checking fails, it outputs $\perp$. Next, it obtains $E K_{A B E}$ and $E K_{S U E}$ by running CP-ABE.Decaps $\left(C_{A B E}\right.$, $\left.S K_{A B E}, P P_{A B E}\right)$ and SUE.Decaps $\left(C H_{S U E}, S K_{S U E}, P P_{S U E}\right)$ respectively. If $E K_{A B E} \neq \perp$ and $E K_{S U E} \neq \perp$, then it outputs a session key $E K=E K_{A B E} \cdot E K_{S U E}$. Otherwise, it outputs $\perp$.

Remark 14. Let $C H_{\mathbb{A}, T}$ and $C H_{\mathbb{A}^{\prime}, T^{\prime}}^{\prime}$ be two valid RS-ABE ciphertext headers with SUE ciphertext headers $C H_{S U E}$ and $C_{S U E}^{\prime}$ respectively. The efficiently computable relation $R\left(\mathrm{CH}_{\mathbb{A}, T}, C H_{\mathbb{A}^{\prime}, T^{\prime}}^{\prime}\right)$ in Remark 11 returns True if $R\left(\mathrm{CH}_{S U E}, \mathrm{CH}_{S U E}^{\prime}\right)=$ True in Remark 8 .

Remark 15. In contrast to CPA secure RS-ABE schemes [21,22,30], our RS-ABE scheme does not provide a ciphertext re-randomization algorithm RandCT. Because of this, the outputted (future) ciphertext header of UpdateCT maybe correlated to the original (past) ciphertext header. Thus, the (past) original ciphertext header should be deleted after running the UpdateCT algorithm to remove the correlation between ciphertext headers.

\subsection{Correctness}

We first show the correctness of the above CP-ABE scheme. Compared to the original CP-ABE scheme of Rouselakis and Waters [29], the above CP-ABE scheme additionally contains elements $u_{B}, h_{B}$ in public parameters and an element $C_{3}$ in a ciphertext header. The validity of $C_{3}$ can be easily checked by using bilinear maps since $C_{3}$ is a DDH tuple. Thus the above CP-ABE scheme is correct since the ciphertext header can pass CP-ABE.VerifyCT and the original CP-ABE scheme is correct.

The correctness of the above RS-ABE scheme can be shown by using the correctness of the CP-ABE scheme, SUE scheme, and CS scheme. Let $S K_{S, u}$ be a private key and $U K_{T^{\prime}, R}$ be an update key. If $u \notin R$, then there are $S K_{A B E}$ in $S K_{S, u}$ and $S K_{S U E}$ in $U K_{T, R}$ that are associated with the same node $v_{i}$ by the correctness of the CS scheme. The decryption key $D K_{S, T^{\prime}}=\left(S K_{A B E}^{\prime}, S K_{S U E}^{\prime}\right)$ is obtained from $S K_{A B E}$ and $S K_{S U E}$ after additional randomization. Note that the master key part of $S K_{A B E}^{\prime}$ is $\gamma_{i}+\delta$ and the master key part of $S K_{S U E}^{\prime}$ is $\alpha-\gamma_{i}-\delta$. Let $C_{\mathbb{A}, T}=\left(C_{A B E}, C H_{S U E}\right)$ be a ciphertext header. If $S \in \mathbb{A}$, then CP-ABE.Decaps can derive a partial session key $E K_{A B E}$ from the correctness of the CP-ABE scheme. If $T \leq T^{\prime}$, then SUE.Decaps can derive a partial session key $E K_{S U E}$ from the correctness of the SUE scheme. By multiplying two partial session keys, we obtain a valid session key since the original master key $\alpha$ can be derived from the master key parts of $\mathrm{ABE}$ and SUE. 


\subsection{Security Analysis}

To prove the TEC-CCA security of the above RS-ABE scheme, we use the $n$-RW1 assumption introduced by Rouselakis and Waters [29]. Rouselakis and Waters proposed an efficient CP-ABE scheme and prove its CPA security under the $n$-RW1 assumption. The definition of $n$-RW1 assumption and the security of the original CP-ABE scheme are given as follows:

Assumption 2 ( $n$-RW1, [29]). Let $\left(p, \mathbb{G}, \mathbb{G}_{T}, e\right)$ be a description of the bilinear group of prime order $p$. Let $g$ be a random generator of $\mathbb{G}$. The $n$-RW1 assumption is that if the challenge tuple

$$
\begin{gathered}
D=( \\
\left\{\left(p, \mathbb{G}, \mathbb{G}_{T}, e\right), g, g^{c},\left\{g^{a^{i}}, g^{d_{j}}, g^{c d_{j}}, g^{a^{i} d_{j}}, g^{a^{i} / d_{j}^{2}}\right\}_{\forall 1 \leq i, j \leq n},\left\{g^{a^{i} / d_{j}}\right\}_{\forall 1 \leq i \leq 2 n, i \neq n+1, \forall 1 \leq j \leq n},\right. \\
\left.\left\{g^{a^{i} d_{j} / d_{j^{\prime}}^{2}}\right\}_{\forall 1 \leq i \leq 2 n, \forall 1 \leq j, j^{\prime} \leq n, j^{\prime} \neq j},\left\{g^{a^{i} c d_{j} / d_{j^{\prime}}}, g^{a^{i} c d_{j} / d_{j^{\prime}}^{2}}\right\}_{\forall 1 \leq i, j, j^{\prime} \leq n, j^{\prime} \neq j}\right) \text { and } Z,
\end{gathered}
$$

are given, no PPT algorithm $\mathcal{A}$ can distinguish $Z=Z_{0}=e(g, g)^{a^{n+1} c}$ from $Z=Z_{1}=e(g, g)^{f}$ with more than a negligible advantage. The advantage of $\mathcal{A}$ is defined as $\operatorname{Adv}_{\mathcal{A}}^{n-R W 1}(\lambda)=\mid \operatorname{Pr}\left[\mathcal{A}\left(D, Z_{0}\right)=0\right]-\operatorname{Pr}\left[\mathcal{A}\left(D, Z_{1}\right)=\right.$ $0] \mid$ where the probability is taken over random choices of $a, c,\left\{d_{j}\right\}_{1 \leq j \leq n}, f \in \mathbb{Z}_{p}$.

Lemma 4.2 ( [29]). The n-RW1 assumption holds in the generic bilinear group model.

Theorem 4.3 ( [29]). The original CP-ABE scheme is selectively secure under chosen plaintext attacks if the $n$-RWI assumption holds where $n$ is the number of columns in the challenge matrix.

To prove the CCA security of the above $\mathrm{CP}-\mathrm{ABE}$ scheme, we use the meta-simulation technique that uses the previous CPA simulator in Theorem 4.3 as a sub-simulator. As pointed by Lee [21], the simulator in Theorem 4.3 cannot be directly used as a sub-simulator in meta-simulation since it sets $\gamma=a^{n+1}+\gamma^{\prime}$ and $w_{A}=g^{a}$. To use this simulator in meta-simulation, we modify the simulator to set $\gamma=a^{n+1}$ and $w_{A}=g^{a} g^{w^{\prime}}$ by selecting a random exponent $w^{\prime}$. Note that this modification is easy. To handle decryption queries of an adversary, we use a variation of the CHK transformation, in which a CPA secure IBE scheme can be converted to a CCA secure PKE scheme [7, 9]. The CCA security of the above CP-ABE scheme is given as follows:

Theorem 4.4. The above $C P-A B E$ scheme is selectively secure under chosen ciphertext attacks if the $n-R W 1$ assumption holds. That is, for any PPT adversary $\mathcal{A}$, we have that $\boldsymbol{A d v}_{\mathcal{A}}^{A B E}(\lambda) \leq \boldsymbol{A d v} \boldsymbol{B}^{n-R W 1}(\lambda)+\boldsymbol{n e g l}(\lambda)$ where $n$ is the number of columns in the challenge matrix.

Proof. Suppose there exists an adversary $\mathcal{A}$ that attacks the above CP-ABE scheme with a non-negligible advantage. A meta-simulator $\mathcal{B}$ that solves the $n$-RW1 assumption using $\mathcal{A}$ is given: a challenge tuple $D=\left(\left(p, \mathbb{G}, \mathbb{G}_{T}, e\right), g, g^{c},\left\{g^{a^{i}}, g^{d_{j}}, g^{c d_{j}}, g^{a^{i} d_{j}}, g^{a^{i} / d_{j}^{2}}\right\},\left\{g^{a^{i} / d_{j}}\right\},\left\{g^{a^{i} d_{j} / d_{j^{\prime}}^{2}}\right\},\left\{g^{a^{i} c d_{j} / d_{j^{\prime}}}, g^{a^{i} c d_{j} / d_{j^{\prime}}^{2}}\right\}\right)$ and $Z$ where $Z=Z_{0}=e(g, g)^{a^{n+1} c}$ or $Z=Z_{1} \in \mathbb{G}_{T}$. Let $\mathcal{B}_{A B E}$ be a modified simulator in the security proof of Theorem 4.3. Then $\mathcal{B}$ that interacts with $\mathcal{A}$ is described as follows:

Init: $\mathcal{A}$ initially submits a challenge access structure $\mathbb{A}^{*} . \mathcal{B}$ first runs $\mathcal{B}_{A B E}$ by giving $D$ and $Z$.

Setup: $\mathcal{B}$ submits $\mathbb{A}^{*}$ to $\mathcal{B}_{A B E}$ and receives $P P_{A B E}=\left(\left(p, \mathbb{G}, \mathbb{G}_{T}, e\right), g, w_{A}, v_{A}, u_{A}, h_{A}, \Lambda=e(g, g)^{a^{n+1}}\right)$. It also requests a challenge ciphertext to $\mathcal{B}_{A B E}$ and receives a challenge ciphertext header $C H_{\mathbb{A}^{*}}=\left(C_{0}^{*},\left\{C_{j, 1}^{*}, C_{j, 2}^{*}, C_{j, 3}^{*}\right\}\right)$ and a challenge session key $E K^{*}$ where $C_{0}^{*}=g^{c}$ and $E K^{*}=Z$. It selects a random index $z$ for a hash function $H^{z}$. It computes $\pi^{*}=H^{z}\left(C_{0}, C_{1,1}, \ldots, C_{l, 3}\right)$ and sets $u_{B}=g^{a^{q}} g^{u_{B}^{\prime}}, h_{B}=\left(g^{a^{q}}\right)^{-\pi^{*}} g^{h_{B}^{\prime}}$ by selecting random exponents $u_{B}^{\prime}, h_{B}^{\prime} \in \mathbb{Z}_{p}$. It implicitly sets $\gamma=a^{n+1}$ and gives the public parameters $P P=$ $\left(\left(p, \mathbb{G}, \mathbb{G}_{T}, e\right), g, w_{A}, v_{A}, u_{A}, h_{A}, z, u_{B}, h_{B}, \Lambda\right)$ to $\mathcal{A}$. 
Query 1: $\mathcal{A}$ adaptively requests a polynomial number of private key and decryption queries. If this is a private key query for a set of attributes $S$, then $\mathcal{B}$ receives a private key $S K_{S}$ from $\mathcal{B}_{A B E}$ by requesting a private key query and gives $S K_{S}$ to $\mathcal{A}$.

If this is a decryption query for a ciphertext header $\mathrm{CH}_{\mathbb{A}}$, then $\mathcal{B}$ proceeds as follows:

1. Let $C H_{\mathbb{A}}=\left(C_{0},\left\{C_{j, 1}, C_{j, 2}, C_{j, 3}\right\}, C_{3}\right)$. It first checks the validity of $C H_{\mathbb{A}}$ by running CP-ABE.VerifyCT $\left(\mathrm{CH}_{\mathbb{A}}, P P\right)$. If the ciphertext header is not valid, then it responds the query with $\perp$. Otherwise, the ciphertext header is valid and formed as $C H_{\mathbb{A}}=\left(C_{0}=g^{t},\left\{C_{j, 1}, C_{j, 2}, C_{j, 3}\right\}, C_{3}=\left(u_{B}^{\pi} h_{B}\right)^{t}\right)$ for some unknown $t \in \mathbb{Z}_{p}$.

2. It calculates $\pi=H^{z}\left(C_{0}, C_{1,1}, \ldots, C_{l, 3}\right)$. If $\pi=\pi^{*}$, then it terminates the simulation with $\mathcal{A}$ and outputs a random bit since it cannot response. If $\pi \neq \pi^{*}$, then it can use the IBE technique of Boneh and Boyen [5] to decrypt the ciphertext header. It sets $D_{0}=\left(g^{a}\right)^{-\left(u_{B}^{\prime} \pi+h_{B}^{\prime}\right) /\left(\pi-\pi^{*}\right)}\left(u_{B}^{\pi} h_{B}\right)^{r^{\prime}}$ and $D_{3}=\left(g^{a}\right)^{-1 /\left(\pi-\pi^{*}\right)} g^{r^{\prime}}$ by selecting a random exponent $r^{\prime} \in \mathbb{Z}_{p}$ and it computes the session key as

$$
E K=e\left(C_{0}, D_{0}\right) \cdot e\left(C_{3}, D_{3}\right)=e\left(g^{t}, g^{a^{n+1}}\left(u_{B}^{\pi} h_{B}\right)^{r}\right) \cdot e\left(\left(u_{B}^{\pi} h_{B}\right)^{t}, g^{-r}\right)=e(g, g)^{a^{n+1} t} .
$$

3. It responses the query with $E K$ as a decapsulated session key.

Challenge: $\mathcal{A}$ requests a challenge ciphertext header and a challenge session key. $\mathcal{B}$ computes $C_{3}^{*}=$ $\left(g^{c}\right)^{u_{B}^{\prime} \pi^{*}+h_{B}^{\prime}}$ since $\left(u_{B}^{\pi^{*}} h_{B}\right)^{c}=\left(g^{a^{n}}\right)^{\left(\pi^{*}-\pi^{*}\right) c}(g)^{\left(u_{B}^{\prime} \pi^{*}+h_{B}^{\prime}\right) c}$. It sets $C H^{*}=\left(C_{0}^{*},\left\{C_{j, 1}^{*}, C_{j, 2}^{*}, C_{j, 3}^{*}\right\}, C_{3}^{*}\right)$ and $E K^{*}$ and gives the challenge tuples to $\mathcal{A}$.

Query 2: Same as Query 1. Note that $\mathcal{A}$ cannot request the decryption query on the challenge ciphertext header $C H^{*}$.

Guess: $\mathcal{A}$ outputs a guess $\mu^{\prime} . \mathcal{B}$ also outputs $\mu^{\prime}$.

To finish the proof, we show that $\mathcal{B}$ can handle decryption queries correctly. The decryption of $\mathcal{B}$ only fails when $\pi=\pi^{*}$ even if $C H_{\mathbb{A}} \neq C H^{*}$. However, the probability of this collision event is negligible since $H^{z}$ is a collision resistant hash function. This completes our proof.

To prove the TEC-CCA security of the above RS-ABE scheme, we also apply the partitioning method by using the meta-simulation technique. As mentioned before, we use the CCA simulator of the CP-ABE scheme and the TEC-CCA simulator of the SUE scheme as sub-simulators to simplify the description of a reduction algorithm (meta-simulator). Compared with the security proof of Lee's RS-ABE scheme in [21], the security proof of our RS-ABE scheme shows the TEC-CCA security instead of the CPA security and proves the security in the selective model instead of the selective revocation list model. An adversary should submit a challenge access structure $\mathbb{A}^{*}$ and challenge time $T^{*}$ before he receives the public parameters in the selective model, whereas the adversary additionally submits a set of revoked users $R L^{*}$ on the challenge time before he receives the public parameters in the selective revocation list model. The selective revocation list model was introduced by Boldyreva et al. [4] to prove the security of their revocable ABE scheme and used in other systems in [21,22, 27].

The main idea of proving the security in the selective model instead of the selective revocation list model is to assigning a user index to a random leaf node in a binary tree and to predicting the number of the adversary's private key queries with the condition $S \in \mathbb{A}^{*}$ where $S$ is a set of attributes in a private key. The meta-simulator can easily generate a private key with $S \notin \mathbb{A}^{*}$ since it can use the $\mathrm{CP}$-ABE simulator by creating an $\mathrm{ABE}$ private key that contains the master key $\alpha$. However, it simply cannot generate a private key with $S \in \mathbb{A}^{*}$ by using the CP-ABE simulator because of the restriction in the CP-ABE security model. Thus 
it creates a private key by setting a random $\gamma_{i}$ in a binary tree as the master key part of CP-ABE. To preserve the consistency of private key and update key generations, the meta-simulator should know the positions of user's private keys with $S \in \mathbb{A}^{*}$ in a binary tree. If the number of private key with $S \in \mathbb{A}^{*}$ is known, then the simulator can handle the private key queries by assigning user indexes to random leaf nodes. The TEC-CCA security of our RS-ABE scheme is described as follows:

Theorem 4.5. The above RS-ABE scheme is selectively secure under time extended challenge chosen ciphertext attacks if the $n-R W 1$ assumption holds. That is, for any PPT adversary $\mathcal{A}$, we have that $\boldsymbol{A d v} v_{\mathcal{A}}^{R S-A B E}(\lambda) \leq$ $q \cdot \boldsymbol{A d} v_{\mathcal{B}}^{n-R W 1}(\lambda)+\boldsymbol{n e g l}(\lambda)$ where $n$ is the number of columns in the challenge matrix and $q$ is the number of private key queries.

Proof. Suppose there exists an adversary $\mathcal{A}$ that attacks the above RS-ABE scheme with a non-negligible advantage. A meta-simulator $\mathcal{B}$ that solves the $n$-RW1 assumption using $\mathcal{A}$ is given: a challenge tuple $D=\left(\left(p, \mathbb{G}, \mathbb{G}_{T}, e\right), g, g^{c},\left\{g^{a^{i}}, g^{d_{j}}, g^{c d_{j}}, g^{a^{i} d_{j}}, g^{a^{i} / d_{j}^{2}}\right\},\left\{g^{a^{i} / d_{j}}\right\},\left\{g^{a^{i} d_{j} / d_{j^{\prime}}^{2}}\right\},\left\{g^{a^{i} c d_{j} / d_{j^{\prime}}}, g^{a^{i} c d_{j} / d_{j^{\prime}}^{2}}\right\}\right)$ and $Z$ where $Z=Z_{0}=e(g, g)^{a^{n+1} c}$ or $Z=Z_{1} \in \mathbb{G}_{T}$. Note that a challenge tuple $D_{D B D H}=\left(g, g^{a}, g^{a^{n}}, g^{c}\right)$ for the DBDH assumption can be easily derived from the challenge tuple $D$ of the $n$-RW1 assumption by setting $b=a^{n}$. Let $\mathcal{B}_{A B E}$ be a modified simulator in the security proof of Theorem 4.4 and $\mathcal{B}_{S U E}$ be a simulator in the security proof of Theorem 3.3. Then $\mathcal{B}$ that interacts with $\mathcal{A}$ is described as follows:

Init: $\mathcal{A}$ initially submits a challenge access structure $\mathbb{A}^{*}$ and challenge time $T^{*} . \mathcal{B}$ first runs $\mathcal{B}_{A B E}$ by giving $D$ and $Z$, and it also runs $\mathcal{B}_{S U E}$ by giving $D_{D B D H}$ and $Z$. Let $q$ be the maximum number of private key queries of $\mathcal{A}$. Let $\tilde{q}$ be the number of private key queries for a set of attributes $S$ and a user index $u$ that satisfy $S \in \mathbb{A}^{*} . \mathcal{B}$ randomly guesses $\tilde{q}$ by selecting a random integer in $\{0, \ldots, q\}$. Note that it can correctly guess $\tilde{q}$ with $1 /(q+1)$ probability. Next, it obtains $\mathcal{B} \mathcal{T}$ by running $\operatorname{CS.Setup}\left(N_{\max }\right)$ where $N_{\max } \geq q$ and assigns a random exponent $\gamma_{i} \in \mathbb{Z}_{p}$ to each node $v_{i} \in \mathcal{B T}$. Let $S N^{*}$ be a set of random leaf nodes in $\mathcal{B} \mathcal{T}$ with $\left|S N^{*}\right|=\tilde{q}$. Recall that $\operatorname{Path}(v)$ is the set of path nodes from the root node to the leaf node $v$. That is, $\operatorname{Path}(v)=\left\{v_{j_{0}}, \ldots, v_{j_{d}}\right\}$ where $v_{j_{0}}$ is the root node and $v_{j_{d}}=v$. Let $\operatorname{SteinerTree}\left(S N^{*}\right)$ be the minimal subtree that connects the root node to all leaf nodes in $S N^{*}$. That is, $\operatorname{SteinerTree}\left(S N^{*}\right)=\bigcup_{v_{j} \in S N^{*}} \operatorname{Path}\left(v_{j}\right)$. Setup: $\mathcal{B}$ submits $\mathbb{A}^{*}$ to $\mathcal{B}_{A B E}$ and receives $P P_{A B E}$, and it submits $T^{*}$ to $\mathcal{B}_{S U E}$ and receives $P P_{S U E}$. It queries an $\mathrm{ABE}$ challenge ciphertext header to $\mathcal{B}_{A B E}$ and receives $C H_{A B E}^{*}$ and $E K_{A B E}^{*}$. It also queries an SUE challenge ciphertext header to $\mathcal{B}_{S U E}$ and receives $C H_{S U E}^{*}$ and $E K_{S U E}^{*}$. It randomizes $\Lambda$ of $P P_{A B E}$ and $\Lambda$ of $P P_{S U E}$ by selecting random exponents $\gamma^{\prime}, \beta^{\prime} \in \mathbb{Z}_{p}$. It implicitly sets $\alpha=a^{n+1}$ and gives the public parameters $P P=\left(P P_{A B E}, P P_{S U E}, \Omega=e\left(g^{a}, g^{a^{n}}\right)\right)$ to $\mathcal{A}$.

Query 1: $\mathcal{A}$ adaptively requests a polynomial number of private key, update key, decryption key, and decryption queries.

If this is a private key query for a set of attributes $S$ and a user index $u$, then $\mathcal{B}$ proceeds as follows:

- Case $S \in \mathbb{A}^{*}$ : In this case, it can creates ABE private keys for path nodes by using $\gamma_{i}$ of $\mathcal{B T}$ for the master key of ABE.

1. If there is an unassigned leaf node in $S N^{*}$, then it randomly assigns a leaf node $v_{u} \in S N^{*}$ to $u$. Otherwise, it aborts the simulation since it failed to guess $\tilde{q}$.

2. It obtains $P V_{u}$ by running CS.Assign $(\mathcal{B T}, u)$. Let $\operatorname{Path}\left(v_{u}\right)=\left\{v_{j_{0}}, \ldots, v_{j_{d}}\right\}$ be the set of nodes that is associated with $P V_{u}$ where $v_{u}$ is the leaf node assigned to $u$ and $v_{j_{d}}=v_{u}$. It retrieves exponents $\left\{\gamma_{j_{0}}, \ldots, \gamma_{j_{d}}\right\}$ from $\mathcal{B} \mathcal{T}$ that are associated with $\operatorname{Path}\left(v_{u}\right)$.

3. For all $v_{j_{k}} \in \operatorname{Path}\left(v_{u}\right)$, it obtains $S K_{A B E, k}$ by running CP-ABE.GenKey $\left(S, \gamma_{j_{k}}, P P_{A B E}\right)$ 
4. It responses the query with the private key $S K_{S, u}=\left(P V_{u}, S K_{A B E, 0}, \ldots, S K_{A B E, d}\right)$.

- Case $S \notin \mathbb{A}^{*}$ : In this case, it can use $\mathcal{B}_{A B E}$ to generate $\mathrm{ABE}$ private keys since $\mathcal{A}$ can only request $S$ such that $S \notin \mathbb{A}^{*}$.

1. It randomly assigns a leaf node $v_{u} \notin S N^{*}$ to $u$.

2. It obtains $P V_{u}$ by running $\mathbf{C S . A s s i g n}(\mathcal{B} \mathcal{T}, u)$. Let $\operatorname{Path}\left(v_{u}\right)=\left\{v_{j_{0}}, \ldots, v_{j_{d}}\right\}$ be the set of nodes that is associated with $P V_{u}$ where $v_{u}$ is the leaf node assigned to $u$ and $v_{j_{d}}=v_{u}$. It retrieves exponents $\left\{\gamma_{j_{0}}, \ldots, \gamma_{j_{d}}\right\}$ from $\mathcal{B} \mathcal{T}$ that are associated with $\operatorname{Path}\left(v_{u}\right)$.

3. It queries an $\mathrm{ABE}$ private key for $S$ to $\mathcal{B}_{A B E}$ and receives $S K_{S}^{\prime}$.

4. For each $v_{j_{k}} \in \operatorname{Path}\left(v_{u}\right)$, it performs the following steps: If $v_{j_{k}} \in \operatorname{Steiner} \operatorname{Tree}\left(S N^{*}\right)$, then it obtains $S K_{A B E, k}$ by running CP-ABE.GenKey $\left(S, \gamma_{j_{k}}, P P_{A B E}\right)$. Otherwise, it obtains $S K_{A B E, k}$ by running CP-ABE.RandKey $\left(S K_{S}^{\prime},-\gamma_{j_{k}}, P P_{A B E}\right)$.

5. It responses the query with the private key $S K_{S, u}=\left(P V_{u}, S K_{A B E, 0}, \ldots, S K_{A B E, d}\right)$.

If this is an update key query for time $T$ and a revoked set $R$, then $\mathcal{B}$ proceeds as follows:

- Case $T<T^{*}$ : In this case, it can use $\mathcal{B}_{S U E}$ to generate SUE private keys since $T<T^{*}$.

1. It obtains $C V_{R}$ by running $\operatorname{CS}$.Cover $(\mathcal{B} \mathcal{T}, R)$. Let $\operatorname{Cover}(R)=\left\{v_{i_{1}}, \ldots, v_{i_{m}}\right\}$ be the set of nodes that is associated with $C V_{R}$. It retrieves exponents $\left\{\gamma_{i_{1}}, \ldots, \gamma_{i_{m}}\right\}$ from $\mathcal{B T}$ that are associated with $\operatorname{Cover}(R)$.

2. It queries an $S U E$ private key for $T$ to $\mathcal{B}_{S U E}$ and receives $S K_{S U E}^{\prime}$.

3. For each $v_{i_{k}} \in \operatorname{Cover}(R)$, it performs the following steps: If $v_{i_{k}} \in \operatorname{SteinerTree}\left(S N^{*}\right)$, then it obtains $S K_{S U E, k}$ by running SUE.RandKey $\left(S K_{S U E}^{\prime},-\gamma_{i_{k}}, P P_{S U E}\right)$. Otherwise, it obtains $S K_{S U E, k}$ by running SUE.GenKey $\left(T, \gamma_{i_{k}}, P P_{S U E}\right)$.

4. It responses the query with the update key $U K_{T, R}=\left(C V_{R}, S K_{S U E, 1}, \ldots, S K_{S U E, m}\right)$.

- Case $T \geq T^{*}$ : In this case, it can create SUE private keys by using $\gamma_{i}$ for the master key of SUE. Let $R^{*}$ be the set of revoked users on the time $T^{*}$ and $R N^{*}$ be the set of revoked leaf nodes on the time $T^{*}$. We first have $S N^{*} \subseteq R N^{*}$ since a revealed private key for $S \in \mathbb{A}^{*}$ should be revoked at some time $T \leq T^{*}$. We also have $\operatorname{Steiner} \operatorname{Tree}\left(R N^{*}\right) \cap \operatorname{Cover}(R)=\emptyset$ since $R^{*} \subseteq R$ if $T \geq T^{*}$. Thus, we have SteinerTree $\left(S N^{*}\right) \cap \operatorname{Cover}(R)=\emptyset$ if $T \geq T^{*}$.

1. If $T=T^{*}$, then it counts the number of leaf nodes $q^{\prime}$ in $R N^{*}$ that satisfy $S \in \mathbb{A}^{*}$ and stops the simulation if $q^{\prime} \neq \tilde{q}$ since it failed to guess $q^{\prime}$.

2. It obtains $C V_{R}$ by running $\operatorname{CS.Cover}(\mathcal{B} \mathcal{T}, R)$. Let $\operatorname{Cover}(R)=\left\{v_{i_{1}}, \ldots, v_{i_{m}}\right\}$ be the set of nodes that is associated with $C V_{R}$. It retrieves exponents $\left\{\gamma_{i_{1}}, \ldots, \gamma_{i_{m}}\right\}$ from $\mathcal{B T}$ that are associated with $\operatorname{Cover}(R)$.

3. For each $v_{i_{k}} \in \operatorname{Cover}(R)$, it obtains $S K_{S U E, k}$ by running $\operatorname{SUE.GenKey}\left(T, \gamma_{i_{k}}, P P_{S U E}\right)$.

4. It responses the query with the update key $U K_{T, R}=\left(C V_{R}, S K_{S U E, 1}, \ldots, S K_{S U E, m}\right)$.

If this is a decryption key query for a set of attributes $S$ and time $T$, then $\mathcal{B}$ proceeds as follows:

- Case $S \notin \mathbb{A}^{*}$ : In this case, it can use $\mathcal{B}_{A B E}$ to generate an $\mathrm{ABE}$ private key since $S \notin \mathbb{A}^{*}$.

1. It queries an $\mathrm{ABE}$ private key for $S$ to $\mathcal{B}_{A B E}$ and receives $S K_{A B E}^{\prime}$. 
2. It selects a random exponent $\delta \in \mathbb{Z}_{p}$ and obtains $S K_{A B E}$ and $S K_{S U E}$ by running CP-ABE.RandKey $\left(S K_{S}^{\prime},-\delta, P P_{A B E}\right)$ and SUE.GenKey $\left(T, \delta, P P_{S U E}\right)$ respectively.

3. It responds the query with the decryption key $D K_{S, T}=\left(S K_{A B E}, S K_{S U E}\right)$.

- Case $S \in \mathbb{A}^{*}$ : In this case, it uses $\mathcal{B}_{S U E}$ to generate an SUE private key since $T<T^{*}$ from the restriction of the security model.

1. It queries an SUE private key for $T$ to $\mathcal{B}_{S U E}$ and receives $S K_{S U E}^{\prime}$.

2. It selects a random exponent $\delta \in \mathbb{Z}_{p}$ and obtains $S K_{A B E}$ and $S K_{S U E}$ by running CP-ABE.GenKey $\left(S, \delta, P P_{A B E}\right)$ and SUE. RandKey $\left(S K_{S U E}^{\prime},-\delta, P P_{S U E}\right)$ respectively.

3. It responds the query with the decryption key $D K_{S, T}=\left(S K_{A B E}, S K_{S U E}\right)$.

If this is a decryption query for a ciphertext header $\mathrm{CH}_{\mathbb{A}, T}=\left(\mathrm{CH}_{A B E}, \mathrm{CH}_{S U E}\right)$, then $\mathcal{B}$ proceeds as follows:

1. Let $C H_{A B E}=\left(C_{0}, \ldots\right)$ and $C H_{S U E}=\left(C H^{(0)}, \ldots\right)$ where $C H^{(0)}=\left(C_{0}^{\prime}, \ldots\right)$. It first checks $C_{0}=C_{0}^{\prime}$. If the check fails, then it responds with $\perp$. It checks the validity of $C H_{A B E}$ and $C H_{S U E}$ by running CP-ABE.VerifyCT $\left(\mathrm{CH}_{A B E}, P P_{A B E}\right)$ and SUE.VerifyCT $\left(\mathrm{CH}_{S U E}, T, P P_{S U E}\right)$ respectively. If two ciphertext headers are not valid, then it responds with $\perp$.

2. If $C H_{A B E} \neq C H_{A B E}^{*}$, then it queries the decryption of $C H_{A B E}$ to $\mathcal{B}_{A B E}$ and receives $E K$. Otherwise, it queries the decryption of $C_{S U E}$ to $\mathcal{B}_{S U E}$ and receives $E K$.

3. It responses the query with $E K$ as a decapsulated session key.

Challenge: $\mathcal{A}$ requests a challenge ciphertext header and a challenge session key for $\mathbb{A}^{*}$ and $T^{*}$. It sets the challenger ciphertext header $C H^{*}=\left(C_{A B E}^{*}, C H_{S U E}^{*}\right)$ and the challenge session key $E K^{*}=Z$. Recall that $C H_{A B E}^{*}$ and $C H_{S U E}^{*}$ were received from sub-simulators $\mathcal{B}_{A B E}$ and $\mathcal{B}_{S U E}$ at the setup stage. It gives $C H^{*}$ and $E K^{*}$ to $\mathcal{A}$.

Query 2: $\mathcal{B}$ handles this phase as the same as the query 1 phase since two sub-simulators $B_{A B E}$ and $B_{S U E}$ can handle this phase as the same as the query 1 phase.

Guess: $\mathcal{A}$ outputs a guess $\mu^{\prime}$. $\mathcal{B}$ also outputs $\mu^{\prime}$.

To finish the proof, we show that the meta-simulator $\mathcal{B}$ correctly handles the queries of $\mathcal{A}$. We first show that private keys are correctly generated. A user with an index $u$ is randomly assigned to a unique leaf node $v_{u}$ in $\mathcal{B} \mathcal{T}$ and the private key of the user consists of $\mathrm{ABE}$ private keys, in which each ABE private key is associated with a node $v_{j_{k}}$ in path nodes from the root node to the leaf node. If $v_{j_{k}} \in \operatorname{SteinerTree}\left(S N^{*}\right)$, an $\mathrm{ABE}$ private key for $v_{j_{k}}$ is generated by setting $\gamma_{j_{k}}$ as the master key of ABE. Otherwise, an ABE private key for $v_{j_{k}}$ is generated by setting $\alpha-\gamma_{j_{k}}$ as the master key of ABE. If $S \in \mathbb{A}^{*}$ where $S$ is the set of attributes in a private key, then $\mathcal{B}$ simply uses $\gamma_{j_{k}}$ stored in $\mathcal{B T}$ although it cannot use $\mathcal{B}_{A B E}$. If $S \notin \mathbb{A}^{*}$, then $\mathcal{B}$ can use $\mathcal{B}_{A B E}$ to generate an $\mathrm{ABE}$ private key with the master key $\alpha$ and then it can later add $-\gamma_{j_{k}}$ in the master key part. Thus, $\mathrm{ABE}$ private keys for private key generation are consistently generated depending on the condition $v_{j_{k}} \in$ SteinerTree $\left(S N^{*}\right)$.

We next show that update keys are correctly generated by presenting that the master key parts of SUE private keys in update keys are consistent with those of ABE private keys in private keys. An update key for $T$ and $R$ consists of SUE private keys, in which each SUE private key is associated with a node $v_{i_{k}}$ in $\operatorname{Cover}(R)$. If $v_{i_{k}} \in \operatorname{SteinerTree}\left(S N^{*}\right)$, then an SUE private key should be generated by setting $\alpha-\gamma_{i_{k}}$ as the master key of SUE. If $v_{i_{k}} \notin$ SteinerTree $\left(S N^{*}\right)$, then an SUE private key should be generated by setting $\gamma_{i_{k}}$ as the master key of SUE. In this case, the consistency of private keys and update keys is preserved since 
the master key $\alpha$ can be derived from the master keys $\alpha-\gamma_{j_{k}}$ and $\gamma_{j_{k}}$ of ABE private key and SUE private key for the same node $v_{j_{k}}$. If $T<T^{*}$, then $\mathcal{B}$ can easily generate SUE private keys since it can use $\mathcal{B}_{S U E}$. If $T \geq T^{*}$, then $\mathcal{B}$ should generate SUE private keys by using $\gamma_{i_{k}}$ stored in $\mathcal{B} \mathcal{T}$ since it cannot use $\mathcal{B}_{S U E}$ from the restriction of SUE. However, there is no problem to generate SUE private keys from the fact that $\operatorname{Cover}(R) \cap$ SteinerTree $\left(S N^{*}\right)=\emptyset$ if $T \geq T^{*}$. Thus, SUE private keys are also consistent with ABE private keys.

Decryption keys are correctly generated since $\mathcal{B}$ can use $\mathcal{B}_{A B E}$ if $S \notin \mathbb{A}^{*}$ or $\mathcal{B}_{S U E}$ if $T<T^{*}$ by the restriction of the security model. The decryption queries are also correctly handled since both $\mathcal{B}_{A B E}$ and $\mathcal{B}_{S U E}$ can handle their own decryption queries with non-negligible probability. The challenge ciphertext header is correctly generated since $C H_{A B E}^{*}$ and $C H_{S U E}^{*}$ are generated by $\mathcal{B}_{A B E}$ and $\mathcal{B}_{S U E}$ respectively and two sub-simulators set $C_{0}=g^{c}$. This completes our proof.

\subsection{Discussions}

Efficiency Analysis. Our RS-ABE scheme is similar to the RS-ABE scheme of Lee [21] in terms of efficiency except that the size of public parameters is increased and pairing operations are added to check the validity of the ciphertext header in our RS-ABE scheme. That is, our RS-ABE scheme has $O\left(\log T_{\max }\right)$ group elements in public parameters, $O\left(\log N_{\max } *|S|\right)$ group elements in a private key, $O\left(r \log \left(N_{\max } / r\right) * \log T_{\max }\right)$ group elements in an update key, and $O\left(l+\log T_{\max }\right)$ group elements in a ciphertext header. The decryption algorithm of SUE requires $O\left(|S|+\log T_{\max }\right)$ pairing operations since the SUE ciphertext verification can be done in $O\left(\log T_{\max }\right)$ pairing operations.

Key-Policy ABE. Our RS-ABE scheme combines the CP-ABE scheme of Rouselakis and Waters [29] and our SUE scheme. There is another kind of ABE, called key-policy ABE (KP-ABE) in which a private key is associated with an access structure and a ciphertext is associated with a set of attributes [17]. We can build a TEC-CCA secure RS-ABE scheme with key-policy by using a CCA secure KP-ABE scheme instead of using a CCA secure $\mathrm{CP}-\mathrm{ABE}$ scheme. For instance, a CCA secure KP-ABE scheme can be derived from the KP-ABE scheme of Rouselakis and Waters [29]. This RS-ABE scheme with key-policy can be proven to be selectively TEC-CCA secure under a $q$-type assumption. We omit the details of the construction and the security proof.

Revocable ABE. A revocable ABE (R-ABE) scheme is an ABE scheme with user revocation. Boldyreva et al. [4] introduced the concept of R-ABE and they presented an R-ABE with key-policy and claimed it security in the selective revocation list model. R-ABE is a special type of RS-ABE since R-ABE only supports user revocation whereas RS-ABE supports both user revocation and ciphertext updating. Thus an R-ABE scheme can be built by combining an ABE scheme, an IBE scheme, and the CS scheme. Note that an SUE scheme for RS-ABE is replaced by an IBE scheme for R-ABE. Boldyreva et al. [4] originally claimed that their R-ABE scheme can be secure in the selective model, but they later corrected it by claiming that their R-ABE scheme is secure in the selective revocation list model, in which an adversary should submit a challenge set of attributes, challenge time, and a set of revoked users on the challenge time. If we use the proof technique of our RS-ABE scheme, we can prove the security of the R-ABE scheme in the selective model instead of the selective revocation list model.

\section{Conclusion}

In this paper, we focused on the CCA security of SUE and RS-ABE since previous SUE and RS-ABE schemes only provide CPA security. In the first part of this work, we defined TEC-CCA security for SUE, 
and then we proposed an efficient SUE scheme and proved its TEC-CCA security under the DBDH assumption. In the second part of this work, we also defined TEC-CCA security for RS-ABE and proposed a TEC-CCA secure RS-ABE scheme in the selective model instead of the selective revocation list model. Our SUE and RS-ABE schemes are the first constructions that achieve (relaxed) CCA security.

There are many interesting problems. The first one is to construct SUE and RS-ABE schemes that support (perfect) ciphertext re-randomization. As mentioned before, encryption schemes with ciphertext re-randomization cannot be proven in TEC-CCA security model since there is no relation between a rerandomized ciphertext and the original one. The second one is to build an RS-ABE scheme that supports a designated entity who can update ciphertexts by using his private key. Note that the designated entity can update ciphertexts, but he cannot decrypt ciphertexts. In cloud storage, we may require the cloud sever only to update ciphertexts instead anyone to update ciphertexts.

\section{References}

[1] Jee Hea An, Yevgeniy Dodis, and Tal Rabin. On the security of joint signature and encryption. In Lars R. Knudsen, editor, Advances in Cryptology - EUROCRYPT 2002, volume 2332 of Lecture Notes in Computer Science, pages 83-107. Springer, 2002.

[2] Michael Backes, Christian Cachin, and Alina Oprea. Secure key-updating for lazy revocation. In Dieter Gollmann, Jan Meier, and Andrei Sabelfeld, editors, Computer Security - ESORICS 2006, volume 4189 of Lecture Notes in Computer Science, pages 327-346. Springer, 2006.

[3] Mihir Bellare, Juan A. Garay, and Tal Rabin. Fast batch verification for modular exponentiation and digital signatures. In Kaisa Nyberg, editor, Advances in Cryptology - EUROCRYPT '98, volume 1403 of Lecture Notes in Computer Science, pages 236-250. Springer, 1998.

[4] Alexandra Boldyreva, Vipul Goyal, and Virendra Kumar. Identity-based encryption with efficient revocation. In Peng Ning, Paul F. Syverson, and Somesh Jha, editors, CCS 2008, pages 417-426. ACM, 2008.

[5] Dan Boneh and Xavier Boyen. Efficient selective identity-based encryption without random oracles. J. Cryptology, 24(4):659-693, 2011.

[6] Dan Boneh, Ran Canetti, Shai Halevi, and Jonathan Katz. Chosen-ciphertext security from identitybased encryption. SIAM J. Comput., 36(5):1301-1328, 2007.

[7] Xavier Boyen, Qixiang Mei, and Brent Waters. Direct chosen ciphertext security from identity-based techniques. In Vijay Atluri, Catherine Meadows, and Ari Juels, editors, ACM Conference on Computer and Communications Security, pages 320-329. ACM, 2005.

[8] Jan Camenisch, Susan Hohenberger, and Michael Østergaard Pedersen. Batch verification of short signatures. J. Cryptology, 25(4):723-747, 2012.

[9] Ran Canetti, Shai Halevi, and Jonathan Katz. Chosen-ciphertext security from identity-based encryption. In Christian Cachin and Jan Camenisch, editors, Advances in Cryptology - EUROCRYPT 2004, volume 3027 of Lecture Notes in Computer Science, pages 207-222. Springer, 2004.

[10] Ran Canetti, Shai Halevi, and Jonathan Katz. A forward-secure public-key encryption scheme. $J$. Cryptology, 20(3):265-294, 2007. 
[11] Ran Canetti and Susan Hohenberger. Chosen-ciphertext secure proxy re-encryption. In Peng Ning, Sabrina De Capitani di Vimercati, and Paul F. Syverson, editors, CCS 2007, pages 185-194. ACM, 2007.

[12] Ran Canetti, Hugo Krawczyk, and Jesper Buus Nielsen. Relaxing chosen-ciphertext security. In Dan Boneh, editor, Advances in Cryptology - CRYPTO 2003, volume 2729 of Lecture Notes in Computer Science, pages 565-582. Springer, 2003.

[13] Ronald Cramer and Victor Shoup. A practical public key cryptosystem provably secure against adaptive chosen ciphertext attack. In Hugo Krawczyk, editor, Advances in Cryptology - CRYPTO '98, volume 1462 of Lecture Notes in Computer Science, pages 13-25. Springer, 1998.

[14] Pratish Datta, Ratna Dutta, and Sourav Mukhopadhyay. Fully secure self-updatable encryption in prime order bilinear groups. In Sherman S. M. Chow, Jan Camenisch, Lucas Chi Kwong Hui, and Siu-Ming Yiu, editors, ISC 2014, volume 8783 of Lecture Notes in Computer Science, pages 1-18. Springer, 2014.

[15] Danny Dolev, Cynthia Dwork, and Moni Naor. Nonmalleable cryptography. SIAM J. Comput., 30(2):391-437, 2000.

[16] Eu-Jin Goh, Hovav Shacham, Nagendra Modadugu, and Dan Boneh. SiRiUS: Securing remote untrusted storage. In NDSS 2003. The Internet Society, 2003.

[17] Vipul Goyal, Omkant Pandey, Amit Sahai, and Brent Waters. Attribute-based encryption for finegrained access control of encrypted data. In Ari Juels, Rebecca N. Wright, and Sabrina De Capitani di Vimercati, editors, ACM Conference on Computer and Communications Security, pages 89-98. ACM, 2006.

[18] Mahesh Kallahalla, Erik Riedel, Ram Swaminathan, Qian Wang, and Kevin Fu. Plutus: Scalable secure file sharing on untrusted storage. In Jeff Chase, editor, FAST 2003. USENIX, 2003.

[19] Seny Kamara and Kristin E. Lauter. Cryptographic cloud storage. In Radu Sion, Reza Curtmola, Sven Dietrich, Aggelos Kiayias, Josep M. Miret, Kazue Sako, and Francesc Sebé, editors, Financial Cryptography and Data Security - FC 2010, volume 6054 of Lecture Notes in Computer Science, pages 136-149. Springer, 2010.

[20] Seny Kamara, Charalampos Papamanthou, and Tom Roeder. CS2: A searchable cryptographic cloud storage system. Technical Report MSR-TR-2011-58, May 2011.

[21] Kwangsu Lee. Self-updatable encryption with short public parameters and its extensions. Des. Codes Cryptogr., 79(1):121-161, 2016.

[22] Kwangsu Lee, Seung Geol Choi, Dong Hoon Lee, Jong Hwan Park, and Moti Yung. Self-updatable encryption: Time constrained access control with hidden attributes and better efficiency. Theor. Comput. Sci., 667:51-92, 2017.

[23] Kwangsu Lee, Dong Hoon Lee, and Jong Hwan Park. Efficient revocable identity-based encryption via subset difference methods. Des. Codes Cryptogr., 85(1):39-76, 2017. 
[24] Benoît Libert and Damien Vergnaud. Adaptive-id secure revocable identity-based encryption. In Marc Fischlin, editor, Topics in Cryptology - CT-RSA 2009, volume 5473 of Lecture Notes in Computer Science, pages 1-15. Springer, 2009.

[25] Dalit Naor, Moni Naor, and Jeffery Lotspiech. Revocation and tracing schemes for stateless receivers. In Joe Kilian, editor, Advances in Cryptology - CRYPTO 2001, volume 2139 of Lecture Notes in Computer Science, pages 41-62. Springer, 2001.

[26] Moni Naor and Moti Yung. Public-key cryptosystems provably secure against chosen ciphertext attacks. In Harriet Ortiz, editor, STOC 1990, pages 427-437. ACM, 1990.

[27] Seunghwan Park, Kwangsu Lee, and Dong Hoon Lee. New constructions of revocable identity-based encryption from multilinear maps. IEEE Trans. Inf. Forensic Secur., 10(8):1564-1577, 2015.

[28] Charles Rackoff and Daniel R. Simon. Non-interactive zero-knowledge proof of knowledge and chosen ciphertext attack. In Joan Feigenbaum, editor, Advances in Cryptology - CRYPTO '91, volume 576 of Lecture Notes in Computer Science, pages 433-444. Springer, 1991.

[29] Yannis Rouselakis and Brent Waters. Practical constructions and new proof methods for large universe attribute-based encryption. In Ahmad-Reza Sadeghi, Virgil D. Gligor, and Moti Yung, editors, ACM Conference on Computer and Communications Security, pages 463-474. ACM, 2013.

[30] Amit Sahai, Hakan Seyalioglu, and Brent Waters. Dynamic credentials and ciphertext delegation for attribute-based encryption. In Reihaneh Safavi-Naini and Ran Canetti, editors, CRYPTO 2012, volume 7417 of Lecture Notes in Computer Science, pages 199-217. Springer, 2012.

[31] Amit Sahai and Brent Waters. Fuzzy identity-based encryption. In Ronald Cramer, editor, Advances in Cryptology - EUROCRYPT 2005, volume 3494 of Lecture Notes in Computer Science, pages 457-473. Springer, 2005.

[32] Jae Hong Seo and Keita Emura. Efficient delegation of key generation and revocation functionalities in identity-based encryption. In Ed Dawson, editor, CT-RSA 2013, volume 7779 of Lecture Notes in Computer Science, pages 343-358. Springer, 2013.

[33] Jae Hong Seo and Keita Emura. Revocable identity-based encryption revisited: Security model and construction. In Kaoru Kurosawa and Goichiro Hanaoka, editors, PKC 2013, volume 7778 of Lecture Notes in Computer Science, pages 216-234. Springer, 2013.

[34] Victor Shoup. A proposal for an ISO standard for public key encryption. Cryptology ePrint Archive, Report 2001/112, 2001. http://eprint.iacr.org/2001/112.

[35] Brent Waters. Ciphertext-policy attribute-based encryption: An expressive, efficient, and provably secure realization. In Dario Catalano, Nelly Fazio, Rosario Gennaro, and Antonio Nicolosi, editors, PKC 2011, volume 6571 of Lecture Notes in Computer Science, pages 53-70. Springer, 2011.

[36] Rui Zhang, Goichiro Hanaoka, Junji Shikata, and Hideki Imai. On the security of multiple encryption or cca-security+cca-security=cca-security? In Feng Bao, Robert H. Deng, and Jianying Zhou, editors, Public-Key Cryptography - PKC 2004, volume 2947 of Lecture Notes in Computer Science, pages 360-374. Springer, 2004. 